\title{
Global Transcriptomic Profiling of the Bone Marrow Stromal Microenvironment during Postnatal Development, Aging, and Inflammation
}

Helbling, Patrick M ; Piñeiro-Yáñez, Elena ; Gerosa, Rahel ; Boettcher, Steffen ; Al-Shahrour, Fátima ; Manz, Markus G ; Nombela-Arrieta, César

\begin{abstract}
Bone marrow (BM) stromal cells provide the regulatory framework for hematopoiesis and contribute to developmental stage-specific niches, such as those preserving hematopoietic stem cells. Despite advances in our understanding of stromal function, little is known about the transcriptional changes that this compartment undergoes throughout lifespan and during adaptation to stress. Using RNA sequencing, we perform transcriptional analyses of four principal stromal subsets, namely CXCL12-abundant reticular, platelet-derived growth factor receptor $(\mathrm{PDGFR})-{ }^{+} \mathrm{Sca} 1^{+}$, sinusoidal, and arterial endothelial cells, from early postnatal, adult, and aged mice. Our data reveal (1) molecular fingerprints defining cellspecific anatomical and functional features, (2) a radical reprogramming of pro-hematopoietic, immune, and matrisomic transcriptional programs during the transition from juvenile stages to adulthood, and (3) the aging-driven progressive upregulation of pro-inflammatory gene expression in stroma. We further demonstrate that transcriptomic pathways elicited in vivo by prototypic microbial molecules are largely recapitulated during aging, thereby supporting the inflammatory basis of age-related adaptations of BM hematopoietic function.
\end{abstract}

DOI: https://doi.org/10.1016/j.celrep.2019.11.004

Posted at the Zurich Open Repository and Archive, University of Zurich

ZORA URL: https://doi.org/10.5167/uzh-180151

Journal Article

Published Version

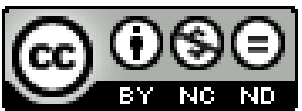

The following work is licensed under a Creative Commons: Attribution-NonCommercial-NoDerivatives 4.0 International (CC BY-NC-ND 4.0) License.

Originally published at:

Helbling, Patrick M; Piñeiro-Yáñez, Elena; Gerosa, Rahel; Boettcher, Steffen; Al-Shahrour, Fátima; Manz, Markus G; Nombela-Arrieta, César (2019). Global Transcriptomic Profiling of the Bone Marrow Stromal Microenvironment during Postnatal Development, Aging, and Inflammation. Cell Reports, 29(10):3313-3330.e4.

DOI: https://doi.org/10.1016/j.celrep.2019.11.004 


\section{Cell Reports}

\section{Global Transcriptomic Profiling of the Bone Marrow Stromal Microenvironment during Postnatal Development, Aging, and Inflammation}

\section{Graphical Abstract}

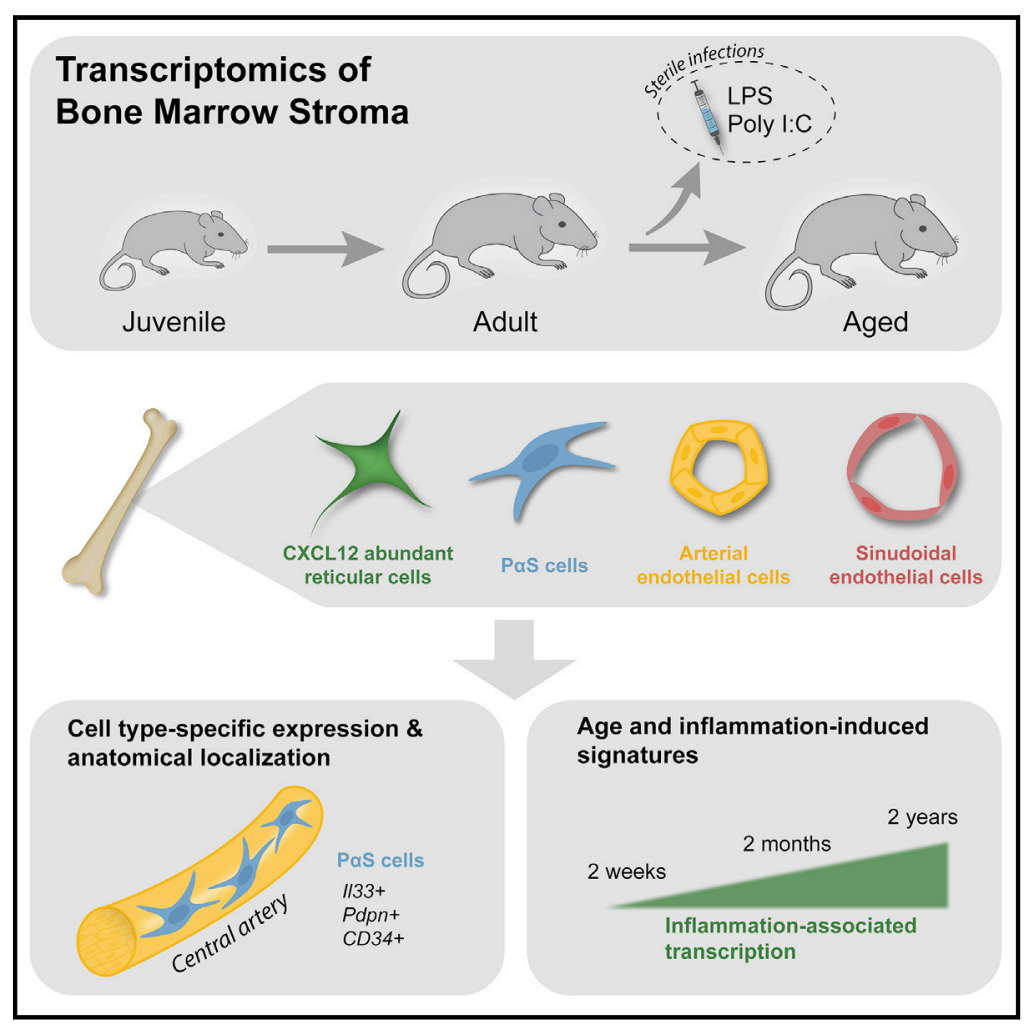

Highlights

- Transcriptional profiling of bone marrow stromal cells throughout postnatal lifespan

- In situ validation of novel stromal markers to map the localization of $\mathrm{PaS}$ cells

- Dynamic remodeling of stromal transcriptome in transition from juvenile to adult stages

- Aging induces prototypical inflammatory transcriptional programs in stromal cells

\section{Authors}

Patrick M. Helbling, Elena Piñeiro-Yáñez, Rahel Gerosa, Steffen Boettcher, Fátima Al-Shahrour, Markus G. Manz, César Nombela-Arrieta

\section{Correspondence}

Cesar.NombelaArrieta@usz.ch

\section{In Brief}

Using RNA sequencing, Helbling et al. analyze the dynamic changes in transcriptional landscape of four major bone marrow stromal cell types, from early postnatal to late aging stages and during responses to sterile infections. The authors reveal the activation of previously unappreciated global and cell-typespecific pro-inflammatory signatures during homeostatic aging. 


\title{
Global Transcriptomic Profiling of the Bone Marrow Stromal Microenvironment during Postnatal Development, Aging, and Inflammation
}

\author{
Patrick M. Helbling, ${ }^{1}$ Elena Piñeiro-Yáñez, ${ }^{2}$ Rahel Gerosa, ${ }^{1}$ Steffen Boettcher, ${ }^{1}$ Fátima Al-Shahrour, ${ }^{2}$ Markus G. Manz,, ${ }^{1,3}$ \\ and César Nombela-Arrieta ${ }^{1,3,4, *}$ \\ ${ }^{1}$ Department of Medical Oncology and Hematology, University Hospital and University of Zurich, 8091 Zurich, Switzerland \\ 2Bioinformatics Unit, Spanish National Cancer Research Center (CNIO), 28029 Madrid, Spain \\ ${ }^{3}$ These authors contributed equally \\ ${ }^{4}$ Lead Contact \\ ${ }^{*}$ Correspondence: Cesar.NombelaArrieta@usz.ch \\ https://doi.org/10.1016/j.celrep.2019.11.004
}

\section{SUMMARY}

Bone marrow (BM) stromal cells provide the regulatory framework for hematopoiesis and contribute to developmental stage-specific niches, such as those preserving hematopoietic stem cells. Despite advances in our understanding of stromal function, little is known about the transcriptional changes that this compartment undergoes throughout lifespan and during adaptation to stress. Using RNA sequencing, we perform transcriptional analyses of four principal stromal subsets, namely CXCL12-abundant reticular, platelet-derived growth factor receptor (PDGFR)$\alpha^{+}$Sca $1^{+}$, sinusoidal, and arterial endothelial cells, from early postnatal, adult, and aged mice. Our data reveal (1) molecular fingerprints defining cell-specific anatomical and functional features, (2) a radical reprogramming of pro-hematopoietic, immune, and matrisomic transcriptional programs during the transition from juvenile stages to adulthood, and (3) the aging-driven progressive upregulation of pro-inflammatory gene expression in stroma. We further demonstrate that transcriptomic pathways elicited in vivo by prototypic microbial molecules are largely recapitulated during aging, thereby supporting the inflammatory basis of age-related adaptations of BM hematopoietic function.

\section{INTRODUCTION}

The continuous production of massive amounts of blood cell lineages is an absolutely essential process, which is centralized in bone marrow (BM) tissues during adulthood and is maintained by hematopoietic stem cells (HSCs) (Eaves, 2015; Nombela-Arrieta and Manz, 2017). Throughout life, BM function quantitatively and qualitatively adjusts to match the fluctuating demands of organisms (Takizawa et al., 2012). During early postnatal development, HSC populations undergo continuous self-renewing proliferation to cope with the rapid growth in blood and marrow volume and the expansion of the hematopoietic system characteristic of these phases. As growth decelerates and definitive body dimensions are reached, HSCs sharply transition to a hibernating state, through a developmentally timed switch, which in mice happens at 3-4 weeks of age (Benz et al., 2012; Bowie et al., 2006). In the absence of overt disease or insults, HSCs will thereafter remain largely quiescent and keep only sporadic cycling activity, sufficient to support the homeostatic maintenance of the HSC pool and hematopoietic production (Bernitz et al., 2016; Nakamura-Ishizu et al., 2014; Takizawa et al., 2011; Wilson et al., 2008). Beyond marked differences in proliferative rates, juvenile and adult HSCs differ in transcriptional regulation and lineage potential (Kim et al., 2007; Ye et al., 2013). Furthermore, compared to adult phases, neonatal BM tissues display important differences in erythropoietic production and mature B cell content (Moscatello et al., 1998; Pihlgren et al., 2001).

Physiological aging also encompasses various alterations in hematopoiesis and immune cell function. Aging leads to a gradual decrease in lympho- and erythropoiesis and a concomitant bias toward myeloid cell production (Geiger et al., 2013). In aged mice, HSCs significantly expand but exhibit deficits in repopulating capacity and homing ability, aberrant lineage differentiation, and activation of intracellular stress pathways (Beerman et al., 2010; de Haan and Lazare, 2018). Several aging-related traits recapitulate those caused by pathogenic or inflammatory challenges (Kovtonyuk et al., 2016). As an example, bacterial infections and immunization adjuvants elicit biased and enhanced production of granulocytes, depletion of $B$ cell progenitor populations, and mobilization of HSCs (Manz and Boettcher, 2014; Ueda et al., 2004, 2005). Viral agents lead to BM hypoplasia, drive monopoiesis, and impair erythropoiesis and B lymphopoiesis (Nombela-Arrieta and Isringhausen, 2017). A hallmark of hematopoietic responses to inflammation is the rapid activation of HSC cycling, which increases their proliferative history and leads to functional deficits (Takizawa et al., 2017; Walter et al., 2015; Zhang et al., 2016). The existing similarities between aging and inflammation-related effects have inspired the inflammaging hypothesis, which associates age-induced degeneration to the progressive emergence of a basal, low-grade inflammatory condition at tissue and systemic levels (Kovtonyuk et al., 2016). 
A

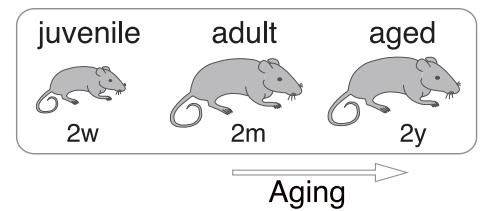

Cell types

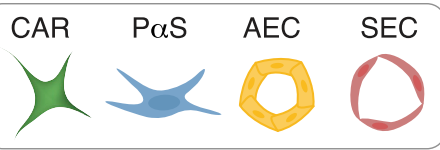

C

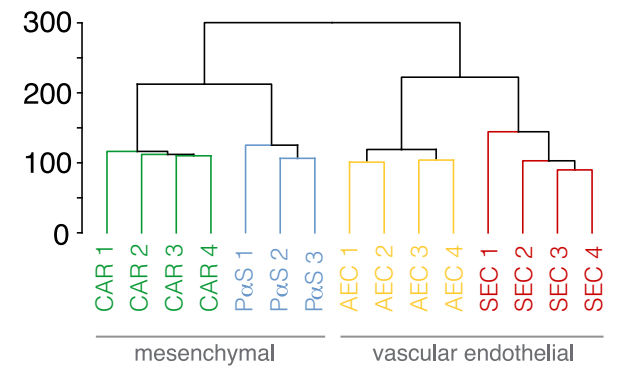

E

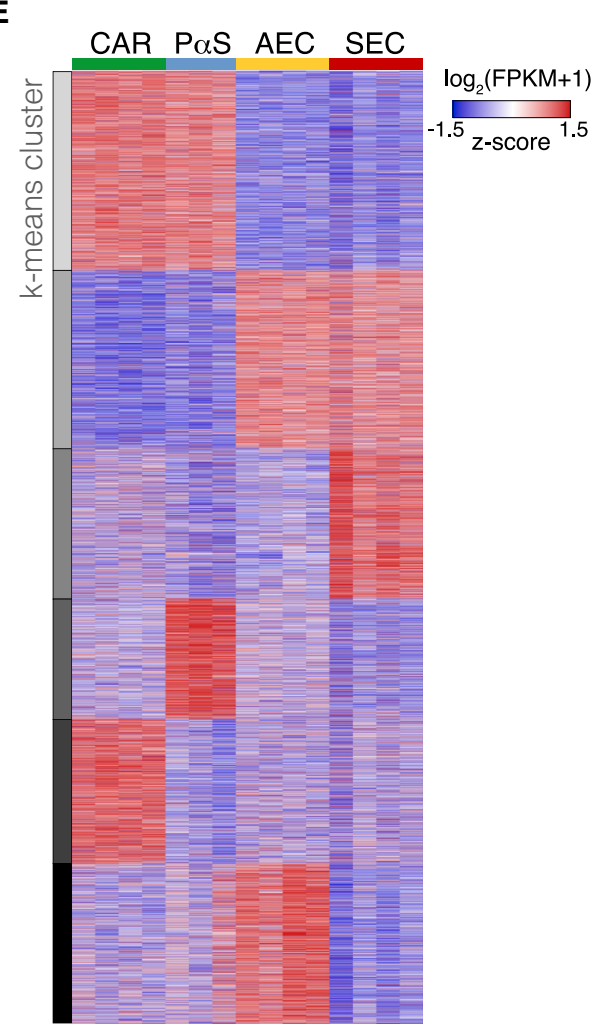

B

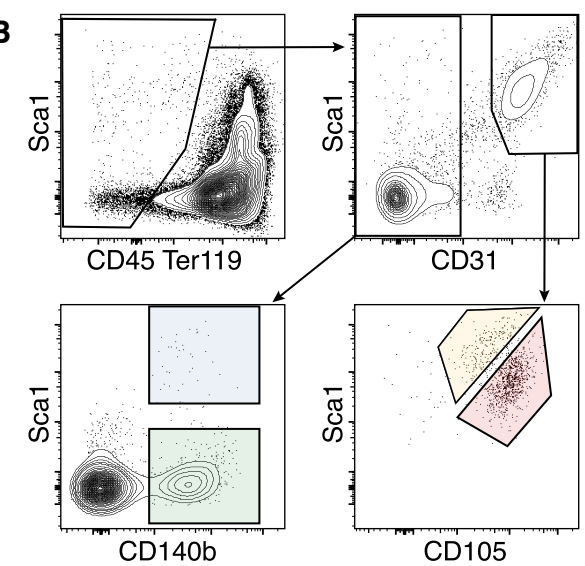

D

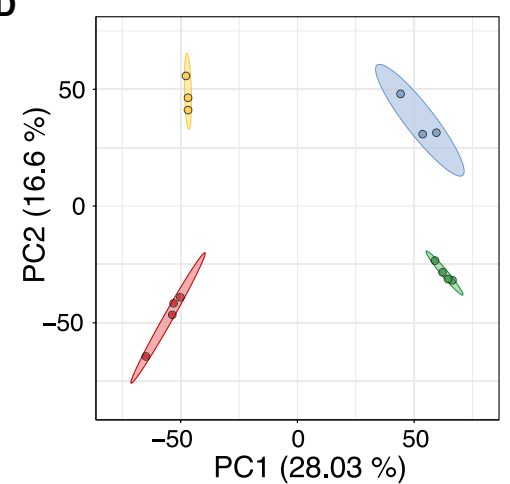

- AEC

- CAR

- PaS

- SEC

$\mathbf{F}$

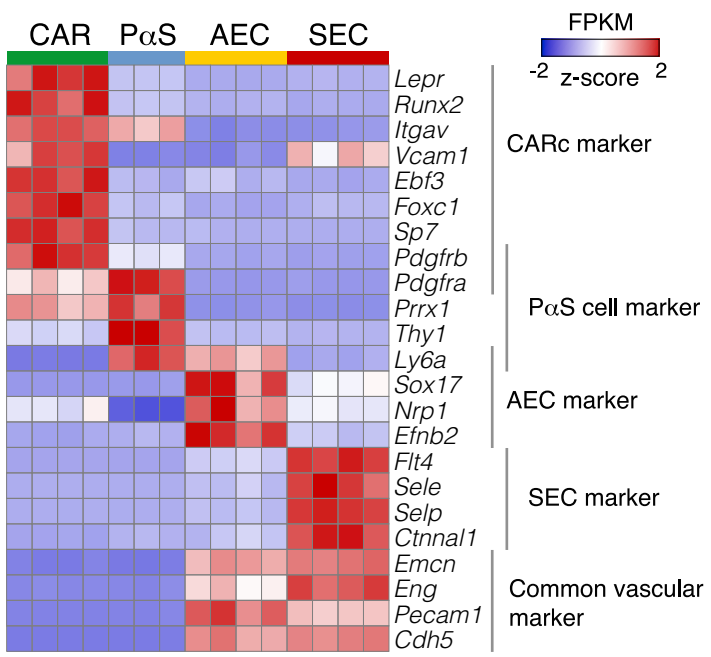


The non-hematopoietic BM stromal compartment modulates hematopoiesis through direct crosstalk and local delivery of regulatory signals (Mercier et al., 2011; Nombela-Arrieta and Manz, 2017). A number of mesenchymal cell subsets have been described, with proven functional roles in the orchestration of homeostatic and reactive hematopoiesis (Kfoury and Scadden, 2015). Thus far, the most extensively studied stromal subset is formed by so-called CXCL12 abundant reticular cells (CARc), which are ubiquitous in adult BM, overlap to a high degree with Leptin receptor-expressing $\left(\mathrm{LepR}^{+}\right)$cells, and are phenotypically defined as $\mathrm{CD}^{-} 5^{-}$Ter119 ${ }^{-} \mathrm{CD} 140 \mathrm{~b}^{+} \mathrm{Sca} 1^{-}$cells. CARc express high levels of stem cell factor (SCF), pleiotrophin (PTN), or interleukin-7 (IL-7) (Cordeiro Gomes et al., 2016; Ding et al., 2012; Himburg et al., 2018) and have been assigned many roles in the direct regulation of HSCs, lympho-, and myelopoiesis (Cordeiro Gomes et al., 2016; Ding et al., 2012; Greenbaum et al., 2013; Omatsu et al., 2010; Tokoyoda et al., 2010). Originally described as a single cellular entity on the basis of the high expression of GFP in Cxc/12-GFP mice (Ara et al., 2003), recent single cell (sc) studies suggest that CARc could potentially comprise a heterogeneous set of osteo- and adipogenic progenitors (Baccin et al., 2019; Baryawno et al., 2019; Tikhonova et al., 2019; Wolock et al., 2019). Additional BM mesenchymal stromal subsets have been characterized in less detail (Kfoury and Scadden, 2015). Among them, platelet-derived growth factor receptors (PDGFR)- $\alpha^{+}$Sca $1^{+}$cells (here designated $\mathrm{P} \alpha \mathrm{Sc}$ ) were initially described on the basis of a phenotypic signature that uniquely differs from that of CARc on the expression of Sca1 (Morikawa et al., 2009). Flow cytometry studies have shown that $\mathrm{P} \alpha \mathrm{Sc}$ are found at highest frequencies in developing bone, but gradually decline to represent a very rare subset in adult BM (Hu et al., 2016; Nusspaumer et al., 2017). Both CARc and PaSc contain CFU-F potential, trilineage differentiation capabilities in vitro, and give rise to osteoblasts, reticular stroma, and adipocytes in vivo (Morikawa et al., 2009; Omatsu et al., 2010). However, the ontogenetic relationships and functional roles in skeletal development and repair of both populations are unclear. Furthermore, although some studies have suggested a potential contribution of $\mathrm{P} \alpha \mathrm{Sc}$ to the regulation of HSCs (Greenbaum et al., 2013; Hu et al., 2016), their anatomical distribution and regulatory roles in hematopoiesis remain largely unexplored.

Endothelial cells are also an integral part of the BM microenvironment. BM microvessels have been divided in three main vascular districts based on structural, topological, and phenotypic criteria (Bixel et al., 2017; Sivaraj and Adams, 2016). Arterial circulation, made up of large, centrally running arteries and arterioles of smaller caliber, gives rise to sinusoidal circulation through so-called transitional vessels (TV) (Kusumbe et al.,
2016). Arterial and sinusoidal endothelial cells (AECs and SECs) are distinguished by specific marker expression (Gomariz et al., 2018; Li et al., 2009; Smith-Berdan et al., 2015), express varying levels of SCF and CXCL12, and regulate different aspects of hematopoiesis (Ding et al., 2012; Xu et al., 2018), from HSC maintenance to maturation of megakaryocytes and late $B$ cell differentiation (Lazzari and Butler, 2018; Stegner et al., 2017).

Although major advances have been made in the understanding of the composition and structure of the stromal compartment in homeostasis, much less is known about the molecular changes that stroma undergoes during the aging process over time and during challenges, and how these may be potentially linked to adaptations of hematopoietic function. Here, we present a longitudinal analysis of the evolution of genome-wide expression profiles of CARc, P $\alpha \mathrm{Sc}, \mathrm{AECs}$, and SECs across three representative stages of postnatal lifespan and during inflammatory responses to prototypical infection-mimicking agents.

\section{RESULTS}

\section{Stromal Cell Isolation, Transcriptional Profiling, and Validation}

We employed previously validated phenotypic signatures to prospectively isolate pure cellular populations of CARc, PaSc, SECs, and AECs using fluorescence-activated cell sorting (FACS) (Figures $1 \mathrm{~A}$ and $1 \mathrm{~B}$ ). Within the endothelial cell population (CD45- Ter119- CD31 ${ }^{\mathrm{hi}}$ ), SECs and AECs were identified as Sca $1^{\text {int }} C D 105^{\text {hi }}$ and Sca $1{ }^{\text {hi }} C D 105^{\text {int }}$, respectively (Figure 1B) (Gomariz et al., 2018). Although TVECs have been recently described (Kusumbe et al., 2014), a molecular signature to reliably distinguish these cells has not been found even using single-cell RNA sequencing (sc-RNA-seq) analyses. Nonetheless, based on immunohistological data, TVECs are rare, they express low/intermediate levels of CD105, and therefore are most likely isolated as AECs in our gating strategy (Gomariz et al., 2018; Itkin et al., 2016; Kusumbe et al., 2014). In turn, mesenchymal CARc and $\mathrm{P} \alpha \mathrm{Sc}$ were sorted from non-hematopoietic (CD45-Ter119-), non-endothelial $\left(\mathrm{CD} 31^{-}\right)$cells as $\mathrm{CD} 140 \mathrm{~b}^{+} \mathrm{Sca} 1^{-}$(CARc) and $\mathrm{CD} 140 \mathrm{~b}^{+} \mathrm{Sca}^{+}$(PaSc) (Figure 1B) (Morikawa et al., 2009). To get a global overview of age-dependent transcriptional dynamics, we isolated all four subsets from 2-week-old ( $2 \mathrm{w}$, juvenile), 2-month-old (2m, adult), and 2-year-old (2y, aged) mice. All populations were phenotypically conserved at different ages (Figure S1A). We sorted 4,000-50,000 cells of each type and condition and performed RNA-seq of at least three independent biological replicates. Transcripts corresponding to non-coding RNAs, pseudogenes, and genes below the detection threshold

Figure 1. Global Transcriptomic Analysis of Juvenile, Adult, and Aged BM Stroma

(A) Schematic overview displaying the four stromal cell types isolated from mice at three different ages investigated in this study. CAR, CXCL12-abundant reticular cells; P $\alpha$ S, CD140b+Sca1 ${ }^{+}$cells; AEC, arterial endothelial cells; SEC, sinusoidal endothelial cells.

(B) Fluorescence activated cell sorting (FACS) gating strategy for BM stromal cell populations (color-coded) in $2 \mathrm{~m}$ mice. Pre-gated for singlets and live cells. (C and D) Unsupervised hierarchical clustering (dendrogram) (C) and principal component analysis (PCA) (D) of the four cell types of $2 \mathrm{~m}$ mice based on expression values (FPKM) of all detected genes.

(E) K-means clustering analysis using all detected genes in stromal cells of $2 \mathrm{~m}$ mice.

(F) Expression values of previously reported stromal cell-type-specific markers. Row $Z$ score normalized.

See also Figure S1. 

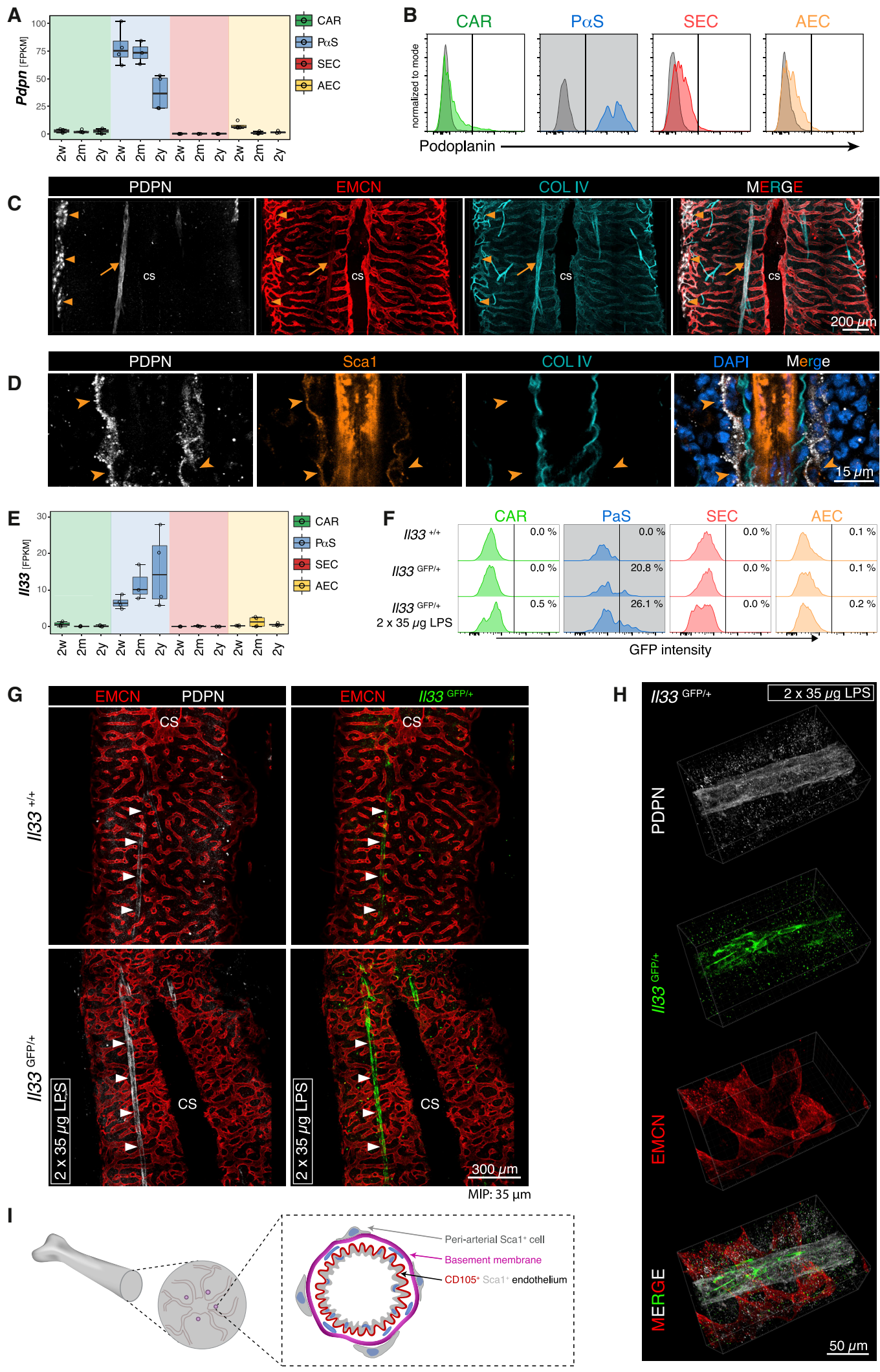
were filtered out, resulting in the identification of a total of 11,595 protein coding genes in all conditions (full list provided in Table S1).

Unsupervised hierarchical clustering and principal component (PCA) analyses revealed very high reproducibility between sample replicates (Figures 1C-1E and S1B). Consistent with their shared identity and developmental origins, endothelial and mesenchymal components separated most from each other, while AECs and SECs, as well as CARc and $\mathrm{P} \alpha \mathrm{Sc}$, respectively, clustered together (Figure 1D). Expression values of previously described type-specific markers, including those employed for their identification and cell sorting, confirmed the robustness of the isolation strategies and cellular identities (Figure 1F). As expected, CARc expressed high levels of Cxcl12, Foxc1, Ebf3, Lepr, Itgva, and Vcam-1. Genes associated to mesenchymal origin (Pdgfra, Pdgfrb, and Prrx1) were commonly expressed by CARc and $\mathrm{P} \alpha \mathrm{Sc}$. Transcripts of prototypical vascular endothelial proteins (Pecam1, Tek, and Cdh5) were exclusively and abundantly detected in SECs and AECs. Arterial-specific Sox17 and Ephb2 were distinctively expressed by AECs, while Selp (P-selectin), Sele (E-selectin), Ctnnal1 (alpha-catulin), and Flt4 (VEGFR-3) were present in the SEC fractions (Acar et al., 2015; Butler et al., 2010; Corada et al., 2013; Gomariz et al., 2018). Notably, the specificity in marker expression was maintained at all time points and cell types consistently clustered throughout developmental stages, indicating preservation of overall cellular identity across lifespan (Figures S1B and S1C).

\section{Cell-Subset Specific Transcriptomic Fingerprints for Phenotypic Detection and Analysis of Spatial Localization}

We mined RNA-seq data from BM from $2 \mathrm{~m}$ mice to uncover genes selectively expressed by distinct subsets in homeostasis. To this end, we established restrictive filtering criteria and focused on genes displaying (1) low/negligible signal (FPKM values <30) in three stromal cell types, and (2) 10-fold increase $\left(\log _{2}\right.$-fold change $[F C]>3.32$, false discovery rate $[F D R] \leq 0.05)$ in the remaining cell type with respect to all other subsets. This yielded transcriptomic fingerprints containing 55, 55, 24, and 38 highly specific genes for CARc, $\mathrm{P} \alpha \mathrm{Sc}, \mathrm{AECs}$, and SECs, respectively (Figure S2A). The CARc signature was dominated by genes associated to osteoblastic development (Runx2, Bglap, Bglap2, Vdr, Sp7, Dmp1, $B m p 3$, and Pth1r), but included genes linked to epithelial to mesenchymal transition (Notch3 and Tbx2), intercellular communication (Cldn10 and Cdh11), and soluble factors of which CARc have not yet been described as a relevant source, such as angiotensinogen (Agt), kininogens 1 and 2 (Kng1 and Kng2), and midkine (Mdk).

The $\mathrm{P} \alpha \mathrm{Sc}$ signature comprised genes characteristic of fibroblastic identity (podoplanin [Pdpn], endosialin [Cd248]) (Figures $2 \mathrm{~A}$ and S2A), other associated to angiogenic regulation (Mfap5 and $T n \times b$ ) and cytokines (//33 and Cc/11). Given the poorly defined anatomical distribution of $\mathrm{P} \alpha \mathrm{Sc}$, we assessed the specificity of some of the identified genes as putative markers to histologically map this population in BM tissues. Flow cytometry analyses confirmed that within CD45- Ter119- CD140b+ cells, expression of $\mathrm{Pdpn}$ was strictly restricted to $\mathrm{P} \alpha \mathrm{Sc}$, which homogeneously expressed high levels of this protein $(98.9 \% \pm 1.1 \%)$ (Figure 2B). Using 3D imaging, we observed a unique subset of $\mathrm{Pdpn}^{+} \mathrm{Sca} 1^{+} \mathrm{CD} 140 \mathrm{~b}^{+}$pericytes, which localized in an adventitial layer surrounding large, central BM arteries but not arterioles (Figures 2C and 2D; Video S1). Of note, bone-lining mature osteoblasts, which had not been included in our study, also expressed Pdpn but could be easily discriminated from $\mathrm{P} \alpha \mathrm{Sc}$ by morphology, endosteal localization, and lack of expression of CD140b (Figures $\mathrm{S} 2 \mathrm{~B}$ and S2C). Expression of $/ / 33$ was also specific for $\mathrm{P} \alpha \mathrm{Sc}$ and strongly increased after stimulation with lipopolysaccharide (LPS) (Figures 2E, 2F, and S2D). In untreated and LPS-treated II33-GFP reporter mice (Oboki et al., 2010), GFP $^{+}$cells were observed wrapped around large arteries (Figures $2 \mathrm{G}$ and $2 \mathrm{H}$; Video S2). These results demonstrate that at least a fraction of cells, categorized as $\mathrm{P} \alpha \mathrm{Sc}$, forms part of a specialized adventitial $\mathrm{Pdpn}^{+} \mathrm{IL}^{+} 3^{+}$cell layer that ensheaths major BM arterial vessels (Figure 2l).

We next focused on selective gene signatures that would enable specific identification of SECs or AECs. Among markers of AECs, we found arterial specification gap junction genes Gja4 and Gja5 (encoding for connexin 37 and connexin 40), the transcription factor Sox13, and the angiocrine factor Jag2. SECspecific genes included endothelial selectins (Selp and Sele), as well as three major scavenger receptors, namely stabilin 1 and 2 (Stab1 and Stab2) and the mannose receptor-C type 1 (Mrc1 also known as CD206, Figure S2A). The characteristic expression of phagocytic molecules in SECs underlie their potential role in mediating clearance of blood-borne particulate substances $(\mathrm{Li}$ et al., 2009). 3D imaging and flow cytometry confirmed that within the BM vascular network, CD206 expression was absent in AECs, weakly emerged in TV, and became very prominent in the entire

Figure 2. Podoplanin and IL-33 Expression Allow for Phenotypic and Anatomical Identification of Periarterial PaSc

(A) RNA-seq expression values (FPKM) for Pdpn (podoplanin).

(B) Flow cytometry analysis of PDPN expression in the four stromal cell types. Isotype controls are displayed as gray histograms.

(C) Immunofluorescence staining of a thick femoral section. PDPN-expressing cells are located close to the endosteal surface (triangle shape) and around large arteries (arrow). Scale bar, $200 \mu \mathrm{m}$.

(D) Higher magnification of a centrally located artery. Arrowheads mark PDPN ${ }^{+}$Sca1 ${ }^{+}$periarterial cells. Scale bar, $15 \mu \mathrm{m}$.

(E) RNA-seq expression values (FPKM) for I/33.

(F) Flow cytometry analysis of GFP expression in BM stromal cells from I/33-GFP reporter mice.

(G) 3D imaging of a femoral diaphysis of untreated and LPS treated //33-GFP mice. Sinusoids (endomucin, red), PDPN (white), and //33-GFP (green). Scale bar, 300 $\mu$ m. (H) Volumetric rendering of a large artery and adjacent $/ / 33-\mathrm{GFP}^{+} \mathrm{PDPN}^{+} \mathrm{P} \alpha \mathrm{Sc}$. Scale bar, $50 \mu \mathrm{m}$.

(I) Schematic depiction of the radial localization of large arteries around the central sinus. Large arteries display a thick collagen $\mathrm{IV}^{+}$basement membrane ensheathed by adventitial layers including P $\alpha$ Sc.

See also Figures S2 and S3. 
A

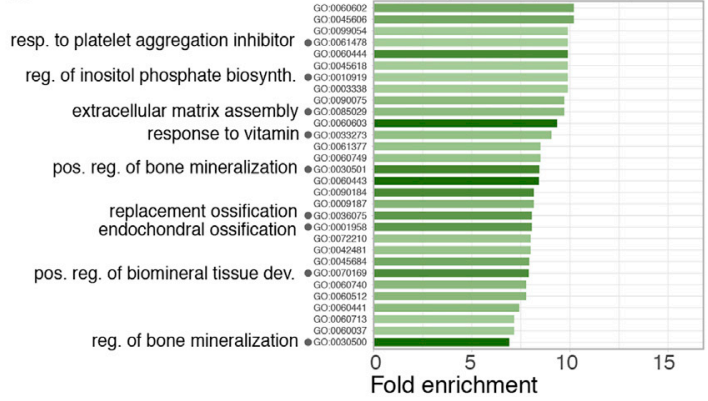

GO molecular function (CAR cells)

t. r. prot. serine/threonine kinase bind. GO molecular function (CAR cells) VEGF receptor bind.
growth factor activity receptor ligand activity

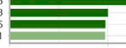

$\begin{array}{lll}0 & 5 & 10 \\ \text { Fold enrichment }\end{array}$

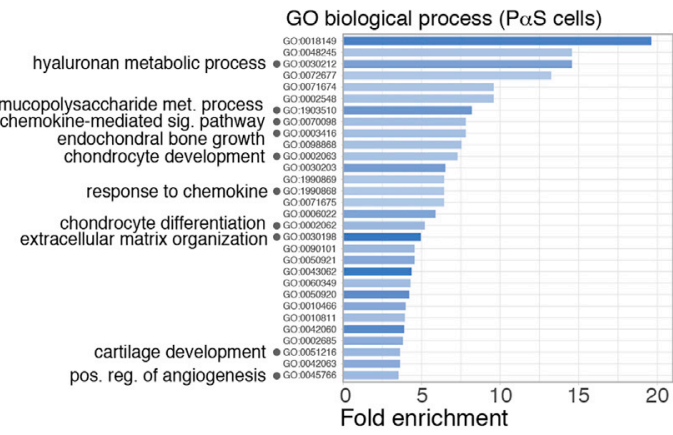

glycosaminoglycan bind. GO molecular function ( $\mathrm{PaS}$ cells) heparin bind.
scavenger receptor activity
collagen bind.

extracellular matrix bind. receptor ligand activity

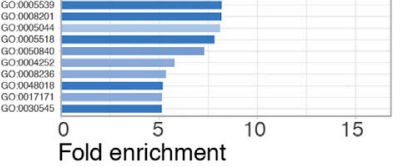

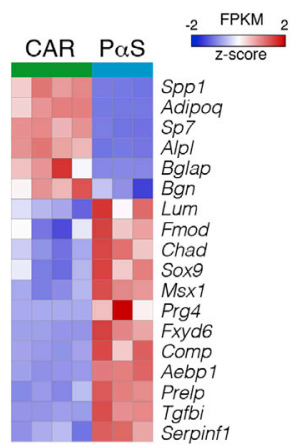

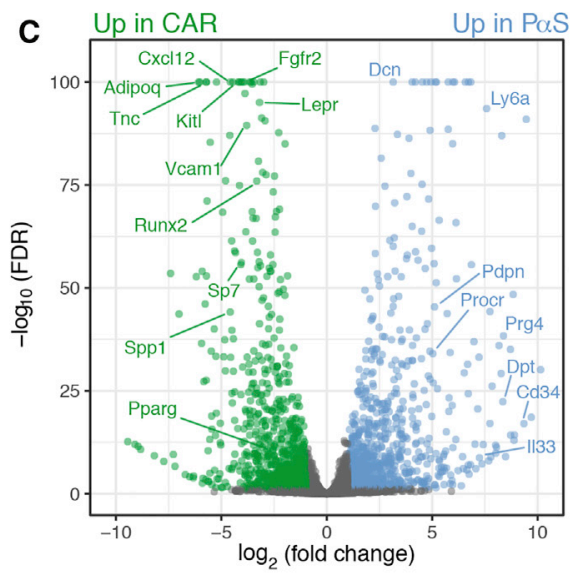

F Cytokines and chemokines

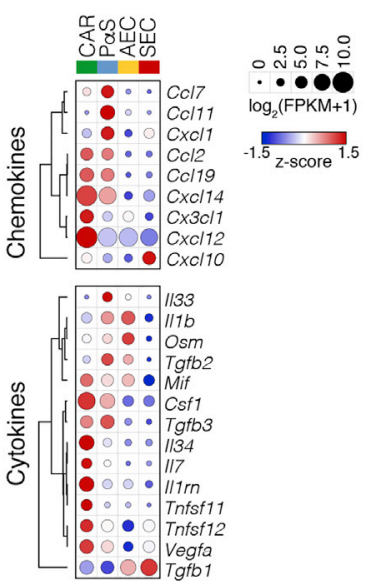

D

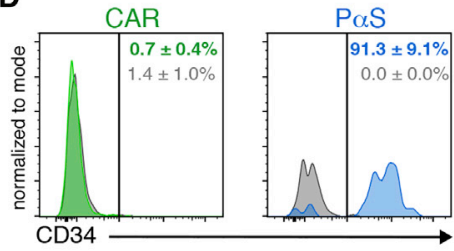

E

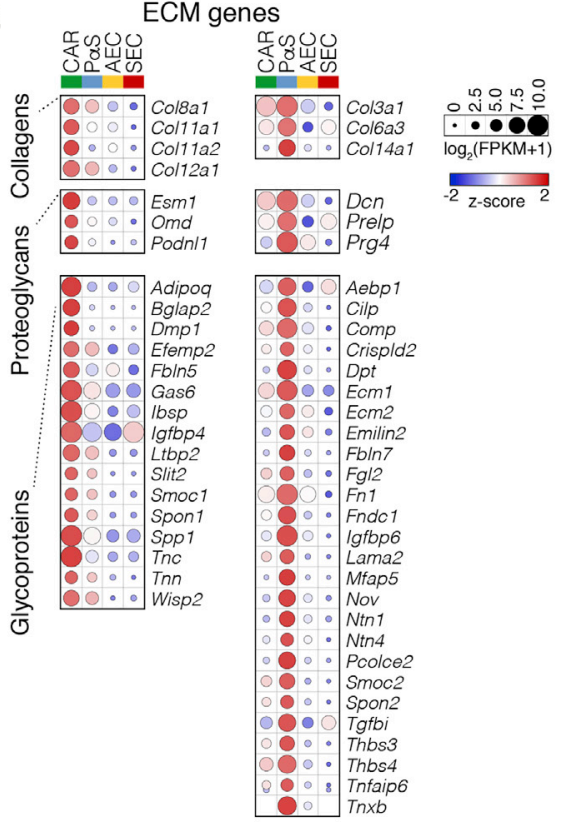

G HSC niche factors

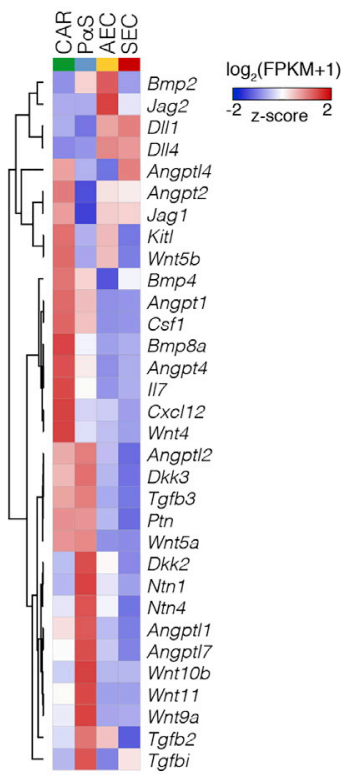

(legend on next page) 
sinusoidal tree (Figures S3A-S3F). CD206 was also expressed in BM macrophages (Figures S3D and S3E).

\section{Transcriptomic Profiles Reveal Mesenchymal Subset- Specific Functions in Adult BM}

To gain insight into the functional, ontogenic, and molecular differences of the two mesenchymal populations, we performed global Gene Ontology (GO) analysis on the entire set of differentially expressed genes (DEGs) $\left(\log _{2} F C>1\right.$, FDR $\left.\leq 0.05\right)$. Consistent with the presence of both osteo- and adipo-primed progenitors, genes upregulated in CARc were overrepresented in biological processes (BPs) related to bone morphogenesis, mineralization, and endochondral ossification (Omatsu et al., 2010; Zhou et al., 2014) (Figures 3A and 3B) and included adipocytic factors such as Adipoq and Pparg (Figures 3B and 3C). In contrast, expression of osteoadipogenic markers was negligible or strongly downregulated in $\mathrm{P} \alpha \mathrm{Sc}$ (Figures $3 \mathrm{~B}$ and $3 \mathrm{C}$ ). GO categories overrepresented in this cell type related to the regulation of cartilage and connective tissue development (Figure 3A). Prototypical genes involved in chondrocyte specification such as Sox9, Fmod, Prg4, Fxyd6, or Lum were expressed at high levels in $\mathrm{P} \alpha \mathrm{Sc}$, which in contrast lacked expression of classical markers of mature chondrocytes (Acan) (Figure 3B) (Boeuf et al., 2008). Compared to CARc, PaSc showed distinctive expression of $\mathrm{Cd} 34$, which was confirmed by flow cytometry and 3D imaging (Figures 3C, 3D, S2F, and S2G).

We found that the transcriptomes of both mesenchymal subpopulations were highly enriched in genes related to extracellular matrix (ECM) (Figure 3A). This was reflected in the abundant expression of a distinctive matrisomic signature of collagens, proteoglycans, and glycoproteins in each mesenchymal subset (Figure 3E). ECM transcripts were also found in EC subpopulations, albeit to a much lower degree. These findings strongly suggest a certain degree of cellular and topographical compartmentalization of the matrisome in the BM, which remains unexplored. CARc transcriptomes were further enriched in gene categories related to cytokine and growth factor activity (Figure 3A), while PaSc, AECs, and SECs subsets showed a much more restricted cytokine expression profile (Figure 3F). In CARc, we detected abundant transcripts of Csf1 and I/34, the two known ligands of the macrophage colony stimulating receptor (M-CSFR), as well as basal expression of the prototypic $\mathrm{T}$ cell chemoattractant $\mathrm{Cc} / 19$, suggesting that CARc, or at least a fraction of this subset, may control basal trafficking of marrow-residing $T$ cell subsets. Paradoxically, CARc not only expressed very high levels of $\mathrm{Cxc} / 12$, but also of $\mathrm{Cxc} / 14$, which has been proposed to function as a partial antagonist of CXCR4 function (Tanegashima et al., 2013). In line with their reported role in monocyte trafficking (Shi et al., 2011), CARc expressed $C c / 2$ and $C x c 3 c / 1$ in homeostasis. Finally, CARc most abundantly expressed a broad range of pro-hematopoietic growth factors (Figure 3G). Beyond Cxcl12, Kitl, II7, or Ptn (Ding et al., 2012; Himburg et al., 2018; Sugiyama et al., 2006), we found substantial expression of Bmp3 and Wnt4 (Louis et al., 2008). In turn, PaSc distinctively expressed certain chemokines and cytokines with known activities in myeloid cell migration and development (Cxc/1, Ccl11, Ccl7, and I/33), the axon guidance protein Netrin1 (Ntn1), and a limited set of relevant pro-hematopoietic genes, which included Wnt antagonists, Dkk2 and Dkk3, as well as Angpt/1, Angpt/2, and Angpt/7 (Xiao, 2015; Xiao et al., 2015).

\section{Major Remodeling of Stromal Transcriptomic Profiles in Early Postnatal Stages}

We next sought to uncover sequential changes in gene expression patterns taking place throughout postnatal life. As expected, we detected substantial transcriptional variations between $2 \mathrm{w}, 2 \mathrm{~m}$, and $2 \mathrm{y}$ mice in all stromal subsets analyzed (Figure 4A). Strikingly, PCA and DEG analyses revealed broader modifications within the short transition from juvenile to adult stages ( 45 days) than those arising in the progression from adult to aged stages (18 months difference) (Figure 4A). To obtain a global view of temporal dynamic changes in transcriptomes we employed Short Time-series Expression Miner (STEM) algorithms. Using this tool, DEGs were grouped in age-dependent expression profiles that were then analyzed for enrichment in GO terms or pathways. In three out of four cell types (CARc, SECs, and AECs), relevant clusters corresponded to DEGs that followed a continuous increase or decrease in expression during aging (designated cluster 1 [c1] and cluster 3 [c3], respectively) or DEGs that were significantly up- or downregulated from $2 \mathrm{w}$ to $2 \mathrm{~m}$, and stabilized thereafter (grouped under $\mathrm{c} 2$ and $\mathrm{c} 4$, respectively) (Figure 4B). The most representative GO terms overrepresented in each cluster are shown in Figure 4B and a complete list is provided in Table S2.

Because functional changes in the BM microenvironment during early postnatal development remain underexplored, we first focused in DEGs contained in c2 and c4, which were exclusively up- or downregulated in the juvenile to adult transition. Unexpectedly, c2 contained genes associated to inflammation and immune activation in all cell types. For instance, genes related to antigen processing and presentation via major

Figure 3. Transcriptomic Comparison of BM Mesenchymal Cells Highlights Functional Differences between CARc and PaSc

(A) Overrepresented GO terms for DEGs in CARc or PaSc compared to the remaining three stromal types (multigroup comparison, FDR $\leq 0.05$, log 2 FC $\geq 2$ ). All displayed GO terms are significant (FDR $\leq 0.05$ ). resp., response; reg., regulation; t.r.prot., transmembrane receptor protein; pos., positive.

(B) Expression of classical osteoblastic or chondrocytic genes in CARc and P $\alpha$ Sc. Columns represent individual biological replicates.

(C) Direct pairwise comparison of DEGs between CARc and P $\alpha$ Sc. Significant DEGs are highlighted in color (FDR $\left.\leq 0.05,-1 \geq \log _{2} F C \geq 1\right)$. Green, significantly upregulated in CARc; blue, significantly upregulated in PaSc.

(D) Flow cytometry analysis of CD34 expression on CARc and PaSc. Isotype controls are shown in gray.

(E) Heatmap of differentially expressed extracellular matrix (ECM) genes ( $F D R \leq 0.05, \log _{2} F C \geq 2$ ) from a multigroup comparison. Only matrisomic $D E G s$ with high expression levels (FPKM $\geq 30$ ) are displayed.

( $F$ and $G$ ) Clustered heatmap showing expression of cytokines and chemokines $(F)$ and HSC niche factors $(G)$ in stromal cells isolated from $2 \mathrm{~m}$ mice. Based on row normalized median expression values. 
A
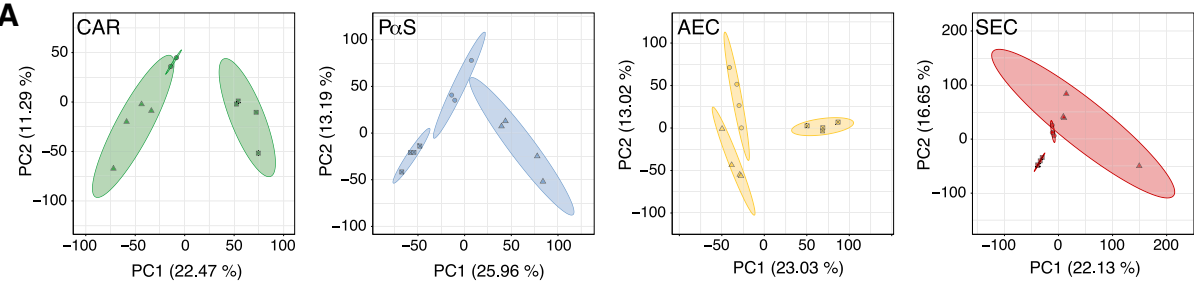

$\triangle 2 \mathrm{w}$

- $2 \mathrm{~m}$

\ $2 \mathrm{y}$
B STEM clustering

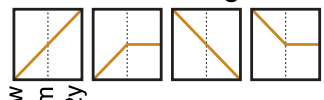

วิ हू ते

\begin{tabular}{l|cccr|}
\multicolumn{5}{c}{ DEGs in cluster } \\
\multicolumn{1}{c}{} & 1 & 2 & 3 & \multicolumn{1}{c}{4} \\
\cline { 2 - 5 } CAR & 364 & 346 & 199 & 199 \\
P $\alpha S$ & 571 & 422 & 275 & 94 \\
AEC & 223 & 484 & 233 & 357 \\
SEC & 230 & 218 & 199 & 185 \\
\cline { 2 - 5 } & & & &
\end{tabular}

C Antigen processing

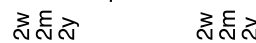

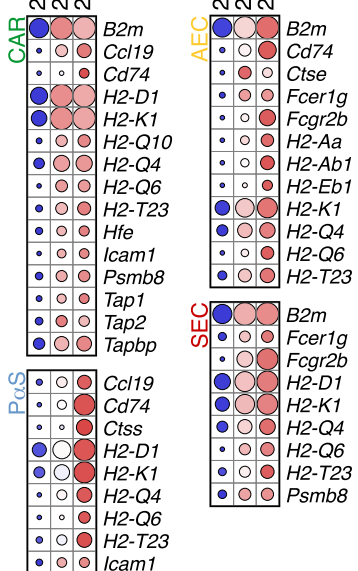

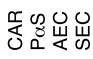

cluster 1 response to chemokine

regulation of acute inflammatory respons antigen processing and presentation regulation of adaptive immune response response to bacterium lymphocyte mediated immunity neutrophil migration granulocyte migration

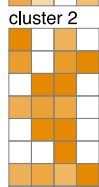

antigen proc. and pres. of peptide antigen response to interferon-beta neutrophil activation cellular extravasation granulocyte activation

granulocyte activation defense response to Gram-neg. bacterium humoral immune response
regulation of coagulation

D ECM organization

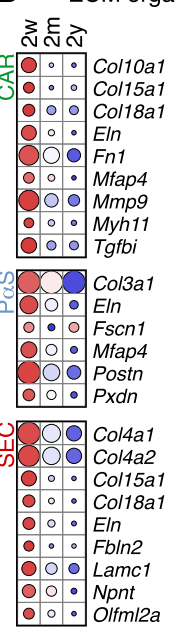

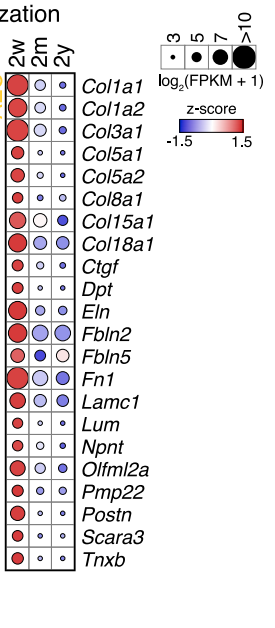

E

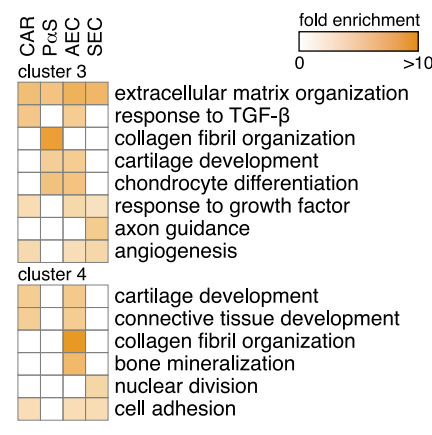

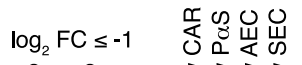

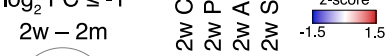

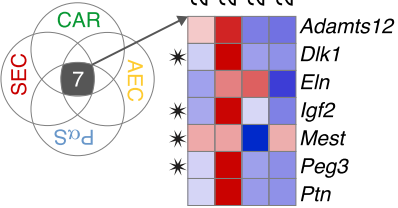

F 츠ำ $P$ aS

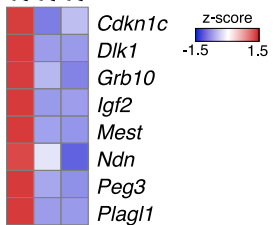

G 2 weeks old 2 months old CD45- CD31

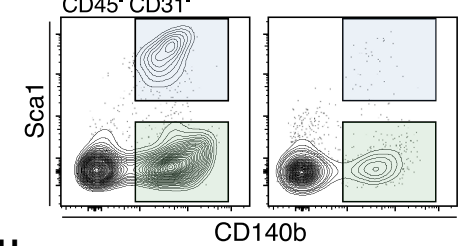

H

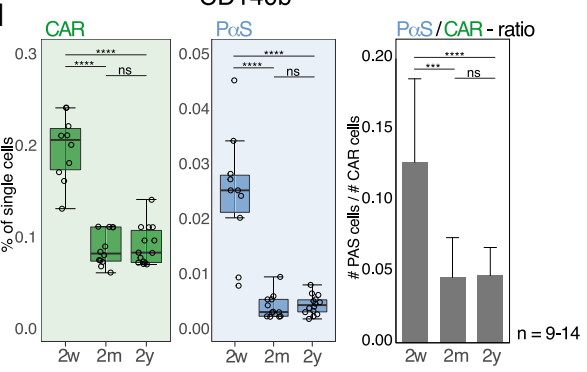

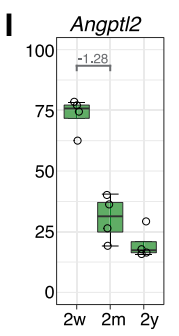
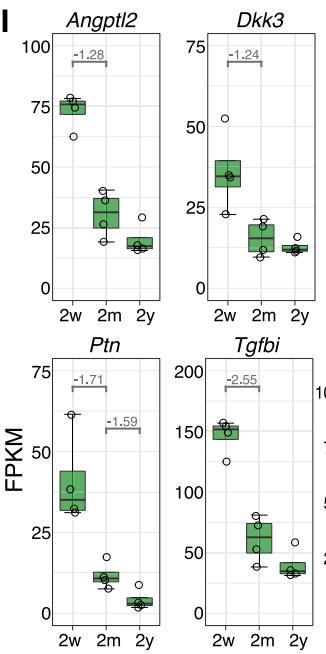

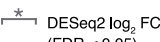
(FDR $\leq 0.05$ )

후 CAR

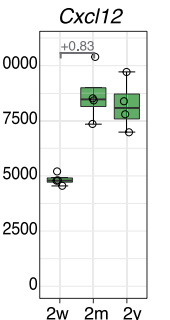


histocompatibility (MHC) class I were highly upregulated in adult compared to juvenile CARc and SECs (Figures 4B and $4 \mathrm{C}$ ). These included classical ( $H 2-D$ and $H 2-K 1, H 2-M 3)$, as well as less characterized non-classical MHC class I molecules $(H 2-Q$ and $H 2-T)$, genes related to peptide transport (B2-m, Tap1, and Tap2) and antigenic presentation in the cell membrane (Figure 4C). Thus, similar to stromal lineages populating lymphoid organs, CARc and SECs are equipped with the molecular repertoire for antigen presentation, and the acquisition of this cellular machinery is developmentally timed to the entry into adulthood. Conversely, DEGs in c4 were overrepresented in BPs related to ECM organization, cartilage and connective tissue development, cell adhesion, responses to transforming growth factor- $\beta$ (TGF$\beta$ ), and angiogenesis. Indeed, a number of genes encoding matrisomic proteins, remodeling enzymes, glycoproteins, and adhesion molecules were conspicuously downregulated in BM mesenchymal cells from $2 \mathrm{w}$ to $2 \mathrm{~m}$ mice (Figure 4D).

We next searched for DEGs that were concurrently downregulated in all stromal lineages in adult compared to juvenile BM. Strikingly, we found a unique common signature of seven genes, which included Ptn, Igf2, and Dlk1, known to play decisive roles in the regulation of self-renewing expansion of adult and embryonic HSCs (Chou and Lodish, 2010; Himburg et al., 2018; Kokkaliaris et al., 2016; Mascarenhas et al., 2009; Thomas et al., 2016). All seven genes displayed by far the highest expression levels in juvenile $\mathrm{P} \alpha \mathrm{Sc}$ compared to other stromal subsets, and the majority (four out of seven, Igf2, DIk1, Peg3, and Mest) belong to a group of genomically imprinted genes, which are monoallelicaly expressed in a parent of origin-specific fashion (Figure 4E). This prompted us to inspect the expression of a core subset of co-regulated imprinted genes, which are highly expressed in multiple somatic stem cells (Berg et al., 2011). We observed that this molecular fingerprint was abundantly expressed in juvenile $\mathrm{PaSc}$ and sharply downregulated during progression to adulthood (Figure $4 \mathrm{~F}$ ). As found in previous studies, $\mathrm{P} \alpha \mathrm{Sc}$ were significantly more abundant in juvenile than in adult BM (Figures $4 \mathrm{G}$ and $4 \mathrm{H})$. Altogether, these results suggest that $\mathrm{P} \alpha \mathrm{Sc}$ display stem cell-like transcriptomic programs, are relatively more abundant in early postnatal BM, and express essential pro-expansive HSC cues, which gradually decline as adulthood is reached. Of note, in this temporal window, we also detected significant downregulation of HSC niche genes in CARc (Angpt/2, Dkk3, and Ptn), whereas expression levels of $C x c / 12$, associated to HSC quiescence and maintenance, followed the inverse trend (Figure 4I).

\section{Increased Pro-inflammatory Signatures in Aged BM Stroma}

STEM analyses revealed that the enrichment in pro-inflammatory signatures was not exclusive of the transition from $2 \mathrm{w}$ to $2 \mathrm{~m}$, but was even more pronounced in $2 \mathrm{y}$ mice (Figure $4 \mathrm{~B}$ ). A total of $274,758,273$, and 159 genes were differentially expressed $\left(\log _{2} F C \geq 1, F D R \leq 0.05\right)$ in aged compared to adult CARc, $\mathrm{P} \alpha \mathrm{Sc}, \mathrm{AECs}$, and SECs, respectively. Using gene set enrichment analysis (GSEA), we detected a general overrepresentation in upregulated genes of numerous inflammatory hallmarks and annotated REACTOME gene-sets (Figures 5A-5C and S4). Of major importance, aged CARc displayed increased expression of $/ / 1 \mathrm{~b}$ and $/ / 6$, cytokines reported to impair HSC self-renewal and enhance proliferation and myeloid bias (Pietras et al., 2016; Schürch et al., 2014; Welner et al., 2015). Among the most prominently upregulated genes were also a number of inflammatory chemokines ( $\mathrm{Cxcl} 2$ and $\mathrm{Cxc} / 5)$, and several members of the complement cascade ( $\mathrm{Cfd}, \mathrm{Cfb}, \mathrm{C} 4 \mathrm{~b}$, and C3) (Figure 5D). These data lend support to the notion that aging gradually results in an enhanced inflammatory milieu, at least partially driven by secretory activity of mesenchymal stromal cells, which drives alterations of BM hematopoiesis. In contrast to what has been proposed, aged CARc did not exhibit changes in expression of crucial pro-hematopoietic factors such as Cxcl12 or Scf. Nonetheless, we did note significant downregulation of Wnt-inhibitory factor (Wif1), Angpt/4, and Npy associated to the modulation of HSC function (Figures $4 \mathrm{I}$ and 5E) (Park et al., 2015; Schumacher et al., 2015; Singh et al., 2017). Moreover, aging was linked to a repression of gene categories associated to matrisomic organization and adhesive interactions, including a number of collagens and enzymes involved in ECM formation (Plod1, Plod2, and Pcolce2) and remodeling (Mmp9 and Mmp11) (Figure 5F), which was strongest in mesenchymal cells but also evident in ECs. Finally, functional annotation of DEGs in ECs during aging uncovered a significant upregulation of gene sets related to translation, posttranscriptional regulation, and RNA and protein metabolism, concomitant with a decrease in angiogenic processes (Figures 5G and S5), which altogether are indicative of prototypic stress responses and could be

Figure 4. Global Ontogenic Transcriptional Changes

(A) PCA of data from time points (2w, 2m, 2y) and cell types (color-coded) based on expression values of all detected genes.

(B) Short time series clustering analysis using STEM. Clustering of DEGs from any inter-condition comparison into pre-defined expression profiles for each cell type. The four significantly overrepresented expression profiles in which DEGs cluster are schematically displayed. Bottom panel: number of DEGs allocated to each individual expression profile (black, FDR $\leq 0.05$; gray, not significant, FDR $\geq 0.05$ ). Heatmaps display fold enrichment of preselected significantly overrepresented GO terms for each profile (full list of GO terms in Table S2).

(C and D) Heatmaps of genes significantly upregulated during the $2 w$ to $2 m$ transition (FDR $\leq 0.05$, $\log _{2} F C \geq 1$ ) belonging to the GO terms "antigen processing and presentation" (C) and "ECM organization" (D). Genes with FPKM $\geq 30$ are depicted.

(E) Overlapping downregulated DEGs (FDR $\leq 0.05, \log _{2} F C \leq-1$ ) during the $2 w$ to $2 m$ transition. Heatmap of the 7 downregulated genes common to all cell types. Asterisks mark genomically imprinted genes.

(F) Heatmap of a selected imprinted gene network (Berg et al., 2011). All heatmaps depict row normalized expression values.

(G) Flow cytometry scatterplot pre-gated for non-hematopoietic, non-endothelial (CD45 ${ }^{-} \mathrm{CD} 31^{-}$) cells at $2 \mathrm{w}$ and $2 \mathrm{~m}$. Color-coded CARc (green) and P $\alpha \mathrm{Sc}$ (blue) gate. (H) Frequencies and calculated ratios of $\mathrm{CARc}$ and $\mathrm{P} \alpha \mathrm{Sc}$ at all experimental time points. Statistics, pairwise $t$ tests. ns, not significant ( $p>0.05)$.

(I) RNA-seq expression values for reported HSC niche factors that significantly change during the $2 \mathrm{w}$ to $2 \mathrm{~m}$ time frame. log $\mathrm{FC}_{2} \mathrm{Falues}$ are shown in gray inserted into the graph. 
A

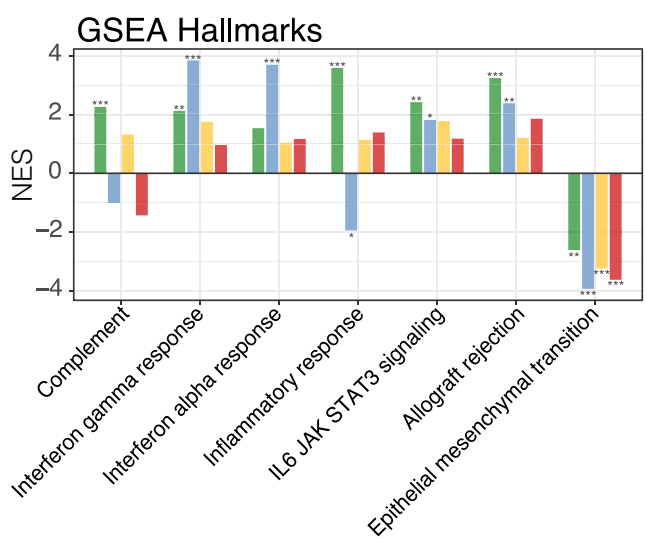

C

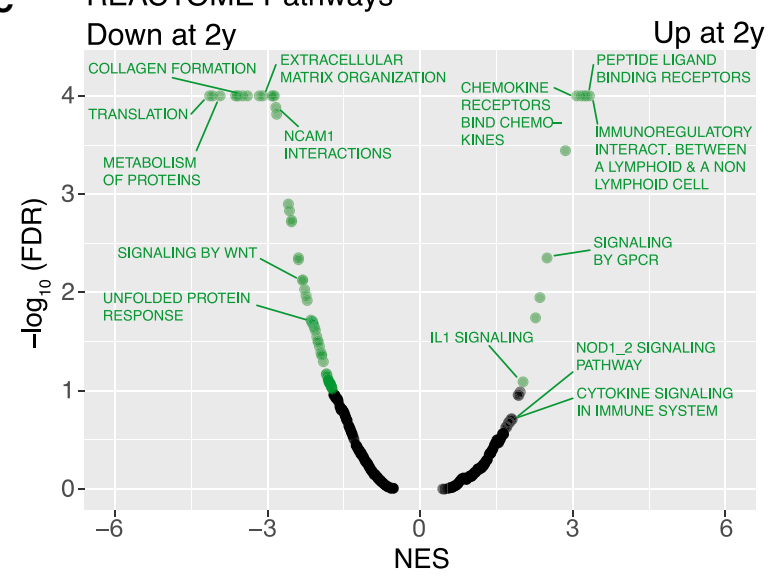

E

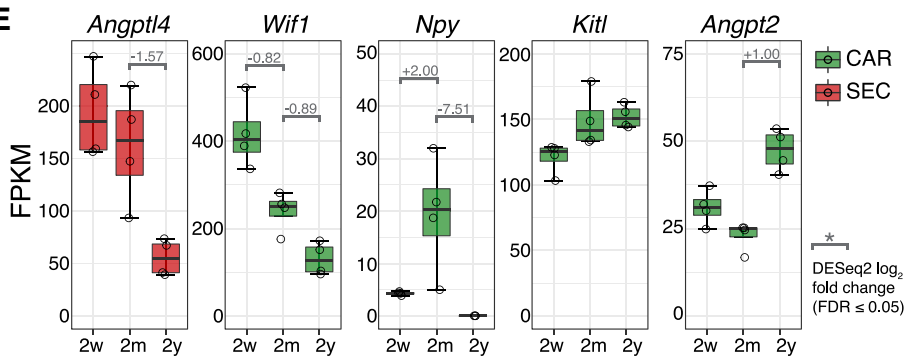

B INFLAMMATORY RESPONSE

CAR $2 y / 2 m$ - PaS $2 y / 2 m$ - SEC $2 y / 2 m$

* FDR $\leq 0.05$ $*$ FDR $\leq 0.005$

$* * *$ FDR $\leq 0.0005$

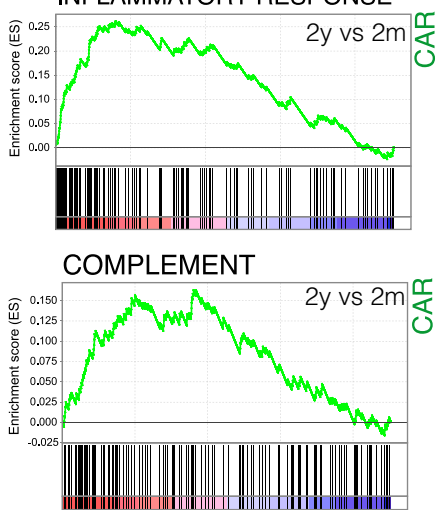

\section{D}
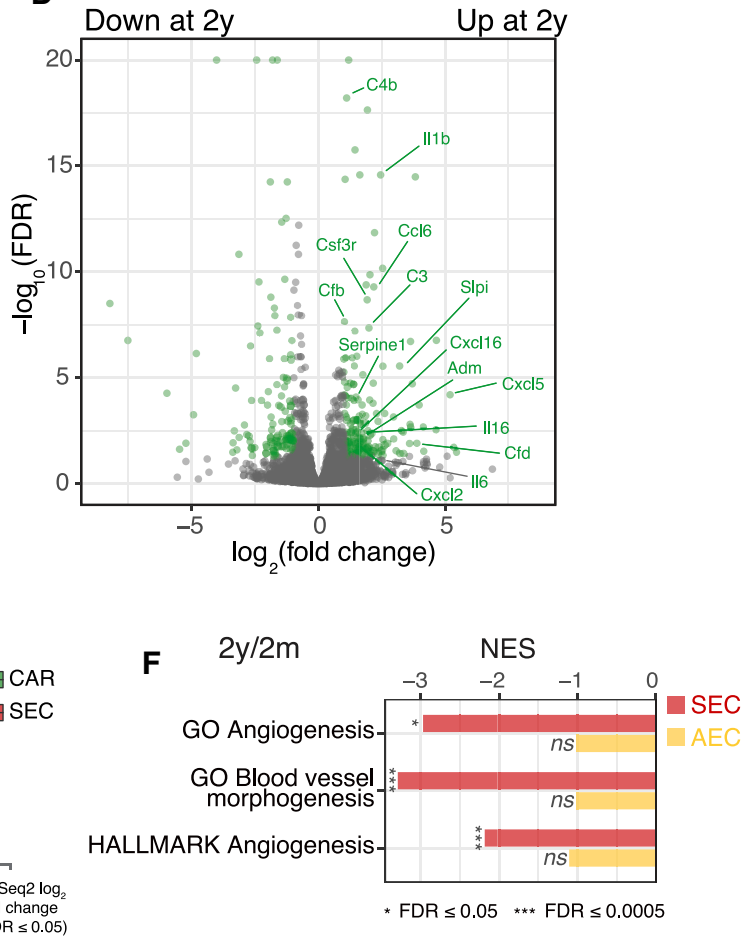

G Differentially expressed matrisome
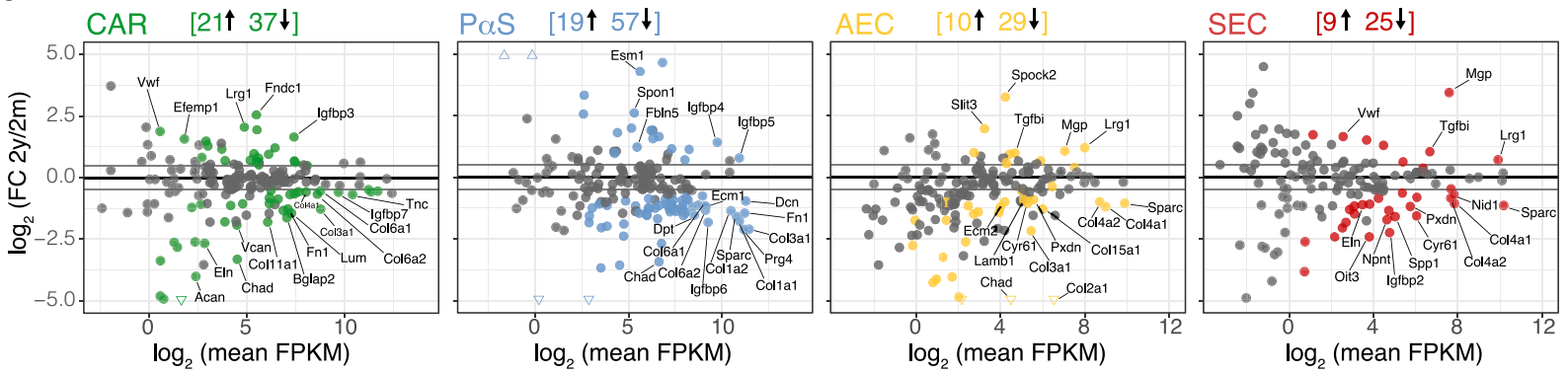

- FDR $\geq 0.05 \quad: F D R<0.05 \quad 177$ core matrisome genes detected 
related to the disrupted arteriolar phenotype observed in aged BM (Maryanovich et al., 2018).

\section{Overlap between Aging and Infection-Induced Transcriptional Changes in BM Stroma}

Having established the pro-inflammatory nature of the aged BM microenvironment, we next compared the magnitude and quality of aging-induced expression changes, with those triggered acutely by administration in adult mice $(2 \mathrm{~m})$ of prototypical bacterial and viral infection-mimicking agents lipopolysaccharide (LPS) or polyinosinic-polycytydilic acid (pl:C). As previously described for HSPCs (Essers et al., 2009), levels of Sca1 were upregulated in all stromal populations during inflammation (Figure S5A). Increases in Sca1 expression led to the shift of a limited, yet significant fraction of SECs into the AEC gate, as well as of CARc into the $\mathrm{P} \alpha \mathrm{Sc}$ gate, which partially contaminated sorted populations and rendered $\mathrm{AEC}$ and $\mathrm{P} \alpha \mathrm{Sc}$ samples unsuitable for further analyses. Thus, we focused subsequent data exploration on CARc and SECs. Administration of both infection mimicking agents elicited acute and profound transcriptional responses in both stromal cell types (Figures 6A-6D). As expected, GSEA identified very strong overrepresentation of DEGs after LPS and pl:C in multiple pathways and hallmark sets associated to inflammatory processes and cytokine responses (Figure 6B). Overall changes in gene expression triggered by infection mimicking agents were more pronounced than those observed in aged BM stroma and strongest in the case of pl:C (Figure 6C). Nonetheless, we noted substantial overlaps in global expression trends and DEGs between LPS, pl:C, and aging when compared to cells isolated from $2 \mathrm{~m}$ mice (Figure 6E). Indeed, out of 274 and $159 \mathrm{DEG}$ upregulated in aged CARc and SECs $\left(\log _{2}\right.$ FC >1, FDR <0.05), $117(43 \%)$ and 56 (35\%) were also found to be significantly increased in LPS and/or pl:C groups (Figure 6E). Among the commonly upregulated genes were chemokines, such as $\mathrm{Cc} / 5, \mathrm{Cc} / 6$, and $\mathrm{Cc} / 19$, as well as Cxcl9, Cxcl10, and Cxcl11, which are ligands of CXCR10 and whose expression is known to be strongly induced by interferon (IFN) signaling. We also found increased expression of $1 / 6$ in CARc, pointing once more to the potential preeminence of this factor in regulating functional aspects of inflammation-driven hematopoiesis. Interestingly, CARc massively increased expression levels of the IL1 receptor antagonist $/ / 1 \mathrm{rn}$, suggesting the activation of a protective mechanism to limit the deleterious effects of IL1 on hematopoietic cells (Figure 6F). In addition, gene sets related to apoptosis, hypoxia, and RNA metabolism were also overrepresented in the transcriptome of stromal cells of mice treated with infection-mimicking agents (Figure 6B).

Other conspicuous changes suggested the potential decline of the hematopoietic supportive function of CARc, through the strong downregulation of key genes involved in extrinsic control of lymphoid, myeloid, and HSPC homeostasis (Cxc/12, Kitl, and II7) (Figure 6G). In contrast, we detected increased expression of Wnt5a, a niche-derived factor required for engraftment of functional HSCs upon transplantation in myeloablated recipients (Schreck et al., 2017). Similar to CARc, expression of pro-hematopoietic genes in SECs was highly reactive to infection-mimicking agents (Figure 6G). Finally, inflammatory stimuli strongly targeted matrisomic gene expression. Major ECM genes, but especially enzymes related to ECM remodeling in CARc and SECs, were differentially expressed in LPS- and pl:C-treated BM with respect to adult controls (Figures $6 \mathrm{H}$ and $\mathrm{S} 5 \mathrm{~B})$. In summary, our results highlight the dynamic responsive nature of BM stromal cells to infections and/or ensuing inflammatory processes and the potential induction of stress gene expression programs in the context of an inflamed and aged BM microenvironment.

\section{DISCUSSION}

Crucial aspects of age-related changes in BM hematopoiesis and HSC function have been elucidated through comprehensive transcriptional and epigenetic studies (Chambers et al., 2007a, 2007b; Gazit et al., 2013; Sun et al., 2014). In contrast, how BM stromal cell function evolves during physiological aging, and whether stromal perturbations correlate with changes in hematopoiesis remains underexplored. In this study, we aimed to reveal novel features of stromal populations and investigate fundamental changes in the molecular make-up of the BM microenvironment throughout postnatal life. We performed bulk RNA-seq of highly pure cell subsets. This approach has the obvious disadvantage of neglecting the heterogeneity that is known to exist within most cell types, which can only be captured using widely employed sc-RNA-seq technologies. Nonetheless, based on recently published data, out of the four populations studied here, CARc are the sole fraction that potentially includes a diverse set of subpopulations, while AECs, SECs, and possibly $\mathrm{P} \alpha \mathrm{Sc}$, constitute largely homogeneous subsets (Baryawno et al., 2019; Tikhonova et al., 2019; Wolock et al., 2019). Therefore, we here opted for population-based transcriptomic analyses, which afford deeper molecular insight and allowed us to study temporal changes in transcriptional landscapes with highest resolution. Perhaps most importantly, our experimental approach required the isolation of BM cellular content following protocols, which involve mechanical disaggregation and cell extraction processes, even though our most recent work demonstrates that substantial fractions of CARc, SECs, and most likely other stromal populations are lost during such

Figure 5. Basal Inflammatory Gene Signature in Aged BM Stroma

(A) GSEA results for selected significant hallmark gene sets. NES, normalized enrichment score.

(B) GSEA enrichment plots for gene sets "inflammatory response" and "complement" in CARc comparing cells isolated from $2 y$ to $2 \mathrm{~m}$ mice (FDR $\leq 0.05$ ).

(C) GSEA results for all REACTOME pathways comparing CARc from $2 \mathrm{y}$ to $2 \mathrm{~m}$ mice. Significant gene sets are displayed in green color (FDR $\leq 0.05$ ).

(D) Differential gene expression results for aged (2y) versus adult (2m) CARc. Significant DEGs are highlighted in green (FDR $\leq 0.05,-1 \geq$ log $_{2}$ FC $\geq 1$ ).

(E) Expression values for selected HSC niche factors. $\log _{2}$ FCs values indicated in gray.

(F) GSEA results for angiogenesis related terms.

(G) Graphs depicting $\log _{2}$ FC of the pairwise comparison $2 y$ versus $2 m$ (y axis) and $\log _{2}$ mean expression values ( $x$ axis) for all core matrisome genes in each cell type. Significant DEGs (FDR $\leq 0.05)$ are highlighted in color. Grey lines mark $\log _{2}$ FC of -1 and 1 as a visual aid. Numbers on top of the plots indicate total numbers of DEGs. 
A
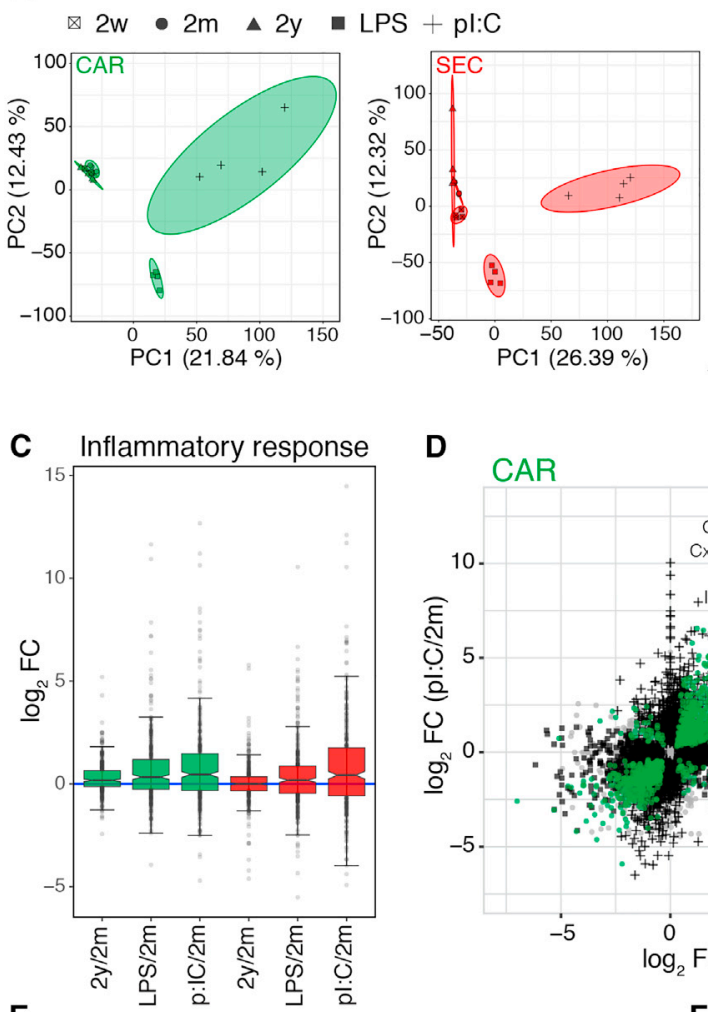

E
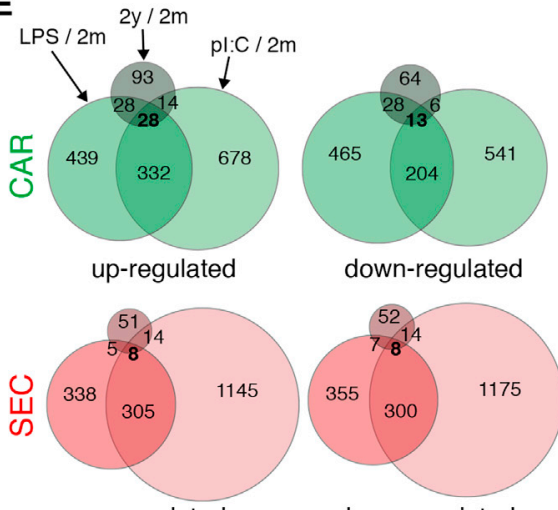

up-regulated

$\mathbf{F}$
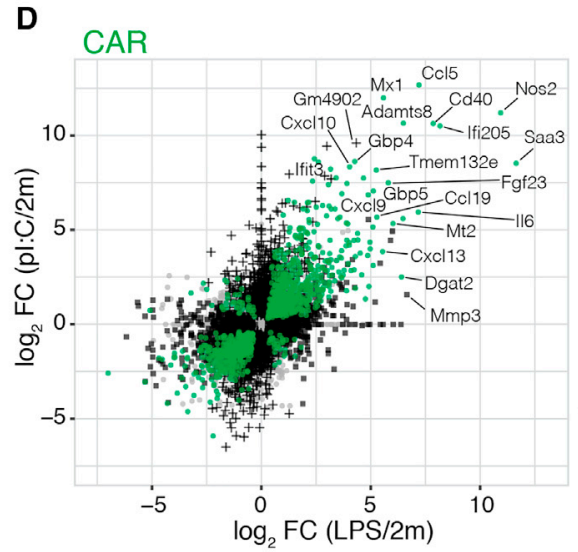

B GSEA Hallmarks

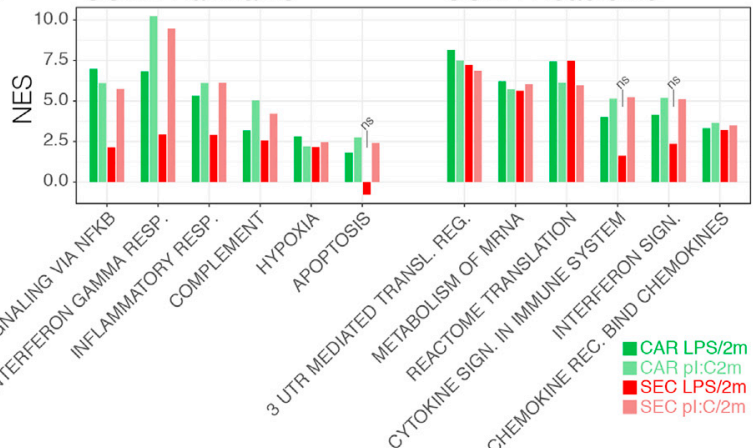

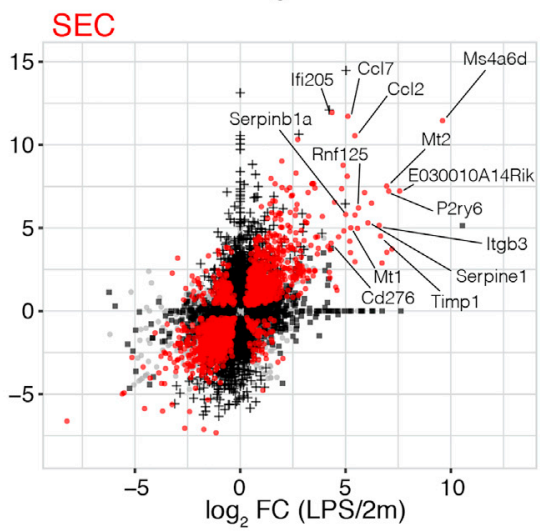
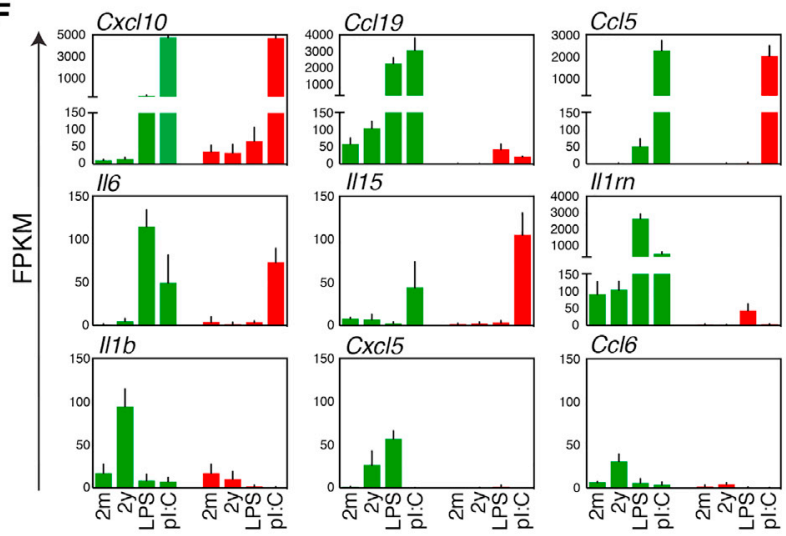

G

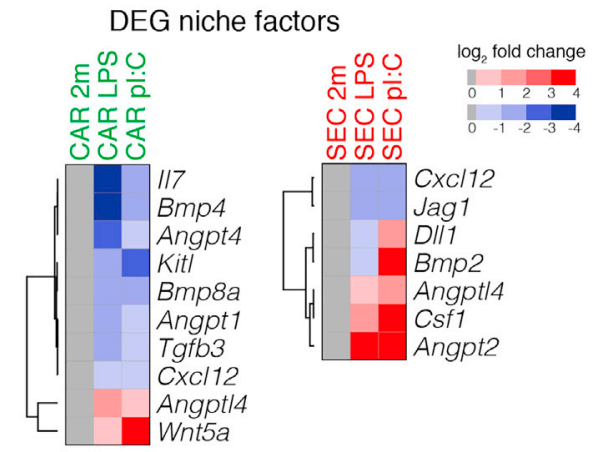

H

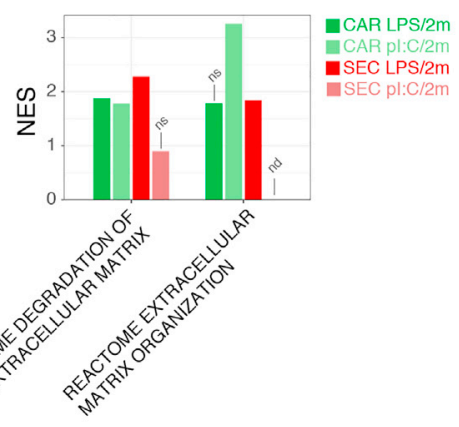


procedures (Gomariz et al., 2018). This is an obvious shortcoming that, nevertheless, equally applies to all studies on BM stroma reported to date and therefore needs to be acknowledged when interpreting results. Having noted this, the datasets presented here (1) provide molecular fingerprints that inform on stromal subset-specific functions and niche affiliations, (2) reveal the previously unappreciated dynamic molecular remodeling of the BM microenvironment during the postnatal window and putative factors supporting self-renewing expansion of HSCs in these phases, and (3) uncover the prominent activation of inflammatory transcriptional programs in stromal cells during aging, which resemble those elicited by infections.

We exploit cell-specific molecular fingerprints to infer unknown functional aspects and unequivocally trace the anatomical localization of $\mathrm{P} \alpha \mathrm{Sc}$. Originally defined as a population enriched in mesenchymal stem cell potential, this cell type has been mostly investigated through the use of in vitro cultures, orthotopic, or systemic transplantation (Hu et al., 2016; Nusspaumer et al., 2017), and its principal biological features remain undefined. Although previous work suggested that $\mathrm{P} \alpha \mathrm{Sc}$ could reside in the proximity of arteries and arterioles (Morikawa et al., 2009; Omatsu et al., 2014), we precisely map PaSc to the external adventitial layer of the large, centrally running femoral arterial branches, which is absent in smaller arterioles. In periarterial bundles, $\mathrm{P} \alpha \mathrm{Sc}$ lie adjacent to, but are distinct from, smooth muscle actin cells and Nestin-GFP cells (not shown) and can be distinguished by expression of Pdpn and IL-33. Altogether, these findings lead us to speculate that $\mathrm{P} \alpha \mathrm{Sc}$ potentially represent a distinct lineage of mesenchymal stromal precursors equivalent to those found in the adventitial cuffs of multiple organs (Sitnik et al., 2016). Recent work suggests a central role of these $\mathrm{CD} 140 \mathrm{~b}^{+} \mathrm{Sca} 1^{+} \mathrm{Pdpn}^{+}$in the maintenance of type 2 innate lymphoid cells (ILC-2) within defined periarterial niches of lung and adipose tissues via expression of IL-33 (Dahlgren et al., 2019; Mahlakõiv et al., 2019; Spallanzani et al., 2019). Given the strong anatomical and molecular analogies between these cells and $\mathrm{P} \alpha \mathrm{Sc}$, their potential contribution to ILC-2 developmental niches in the BM deserves to be explored. Beyond this, although $\mathrm{P} \alpha \mathrm{Sc}$ have been suggested to contribute a critical fraction of CXCL12 production for HSC maintenance (Greenbaum et al., 2013), whether and to what extent this subset influences early stages of hematopoiesis is unclear. We find that in adult mice, $\mathrm{P} \alpha \mathrm{Sc}$ express a distinct set of key HSC pro-expansive factors such as Angpt/2 and Angpt/4. Thus, given their restricted spatial localization and cytokine expression profile, we anticipate that this cell type fulfills unknown functions in he- matopoietic control, which need to be addressed through the development of tailored models allowing in vivo targeting and lineage tracing of $\mathrm{P} \alpha \mathrm{Sc}$ cells. The distinctive $\mathrm{P} \alpha \mathrm{Sc}$ gene signature identified here could prove valuable for the rational design of such experimental tools.

Unlike $\mathrm{P} \alpha \mathrm{Sc}$, a large number of studies have exposed multiple functional facets of CARc. Our data suggest that CARc, as a whole, achieve this remarkable functional pleiotropism through expression of a large plethora of different chemokines and cytokines that potentially regulate not only ontogeny but also trafficking of myeloid and lymphoid populations and immune function of the BM during homeostasis. For instance, CARc abundantly express $/ / 34$ and Csf1, suggesting yet another previously unappreciated function of this cell type in controlling macrophage development and maintenance. These data reinforce the existence of an intimate bidirectional functional relationship between CARc and macrophage networks (Casanova-Acebes et al., 2013; Chow et al., 2011). Future investigations will shed light on whether this, as well as the multiple other jobs of CARc in hematopoietic regulation are independently performed by the recently described CARc subtypes.

While much attention has been cast on the study of aging and BM function, the mechanisms underlying the defining features of juvenile hematopoiesis remain underexplored. Neonatal BM tissues are comparatively more permissive to engraftment of embryonic HSCs than adult counterparts, which points to the supply of unique extrinsic signals by the BM microenvironment at these stages (Arora et al., 2014). In line with this, our data demonstrate that despite conserving phenotypic traits, the four stromal cell types investigated display substantial modifications in their transcriptomes with respect to adult stroma. These differences, which could be imposed by the marked tissue remodeling activity during organ growth, may also be linked to the cellextrinsic orchestration of continuous HSC proliferation in the postnatal window. Remarkably, although embryonic emergence and production of HSCs depend on low-grade pro-inflammatory cues in hematopoietic tissues (Espin-Palazon et al., 2018; He et al., 2015; Sawamiphak et al., 2014; Trompouki, 2016), we find that stromal cells of juvenile BM are almost completely devoid of expression of all inflammatory mediators, MHC class I molecules, and IFN responsive genes, whose expression is abruptly turned on as adulthood is reached. Although we cannot rule out that at this point basal proinflammatory signals are provided by BM-resident innate immune cells, our data strongly suggest that postnatal HSC expansion most likely takes place in a virtually sterile BM milieu. We hypothesize that the

Figure 6. LPS and pl:C Induce Massive Inflammation-Associated Transcriptional Changes

(A) PCA of all conditions including pl:C and LPS for CARc and SECs.

(B) Selection of GSEA results for LPS and pl:C treatment in CARc and SECs.

(C) Log $_{2}$ FC values of all genes belonging to the GO category inflammatory response for CARc (green) and SECs (red).

(D) Scatterplot displaying $\log _{2} \mathrm{FC}$ values of all genes in CARc and SECs in LPS ( $\mathrm{x}$ axis) and pl:C (y axis) treatments compared to adult BM. Significant DEGs (FDR $\leq 0.05)$ in one condition are shown as a black plus sign $(\mathrm{pl}: \mathrm{C} / 2 \mathrm{~m})$ or black square $(\mathrm{LPS} / 2 \mathrm{~m})$, genes that are significant in both inflammatory conditions are displayed as colored dots (green or red) and not significant genes are shown in gray. Selected significant DEGs with high $\log _{2}$ FCs are highlighted.

(E) Venn diagrams of DEGs (FDR $\leq 0.05, \log _{2} F C \geq 1$, or $\log _{2} F C \leq-1$ ) for $2 y$, LPS, and pl:C treated with respect to $2 \mathrm{~m} \mathrm{BM}$.

(F) Expression of selected differentially expressed cytokines and chemokines.

(G) Heatmap of DEGs involved in hematopoietic niche regulation displaying color-coded $\log _{2}$ FCs (LPS versus $2 m$ and pl:C versus $2 m$ ) for CARc and SECs. All shown genes are significant (FDR $\leq 0.05)$.

(H) GSEA results for significant REACTOME gene sets related to extracellular matrix (FDR $\leq 0.05$ ). ns, not significant (FDR $\geq 0.05$ ); nd, not determined. 
non-inflammatory nature of juvenile BM tissues could represent a safeguard mechanism protecting HSCs from the deleterious consequences of uninterrupted proliferation and increased cumulative divisional history observed in inflammation-induced HSC cycling (Takizawa et al., 2017; Walter et al., 2015). In fact, little is known to date on the specific niche-derived signals that promote self-renewing expansion during juvenile development. We found that $\mathrm{P} \alpha \mathrm{Sc}$ in these stages express genes with reported properties in HSC expansion, which are rapidly downregulated during the transition to adulthood. Among them, Igf2 encodes for a mitogenic factor, which is produced by putative niche cell types of the fetal liver (Chou and Lodish, 2010; Mascarenhas et al., 2009; Thomas et al., 2016; Wu et al., 2008). Deregulation of the IGF2-IGFR1 signaling axis in adult BM impairs HSC quiescence, leading to uncontrolled proliferation and exhaustion of the HSC compartment (Venkatraman et al., 2013). Juvenile PaSc also expressed high levels of Dlk1, Dpt, and Ptn, all of which have been assigned HSC supportive activity in vitro and/or in vivo (Kokkaliaris et al., 2016; Wu et al., 2008). Given these striking transcriptomic features and their abundance in juvenile BM, PaSc could potentially be the source of developmental-stagespecific cues driving temporary HSC expansion in postnatal phases.

Our work further sheds light on the fundamental impact of aging on stromal cell biology and BM hematopoiesis. Unexpectedly, we did not detect major changes in CARc expression of classical factors regulating hematopoiesis. However, we noted the emergence of a strong inflammatory signature in the stromal compartment, and particularly in mesenchymal cells, which is in line with previous findings on thymic stroma (Ki et al., 2014). Among multiple cytokine-related genes, expression of $/ 11 \mathrm{~b}$ was strongly increased in CARc from aged mice. Given its recently reported activities in trained immunity, promotion of myelopoiesis, and restriction of self-renewal of HSCs, stromal derived IL-1 is a prime niche candidate for driving age-related HSC deficits and clonal hematopoiesis (Chavakis et al., 2019; Pietras et al., 2016). The induction of a basal inflammatory context is also reflected in the upregulation of multiple components of the complement system, which so far had been associated to HSPC mobilization to peripheral tissues during stress hematopoiesis (Ratajczak et al., 2010). Overexpression of complement genes is a hallmark of aging tissues and abnormal activation of this system has been linked to the emergence of degenerative diseases and cancer (de Magalhães et al., 2009; McGeer et al., 2005; Naito et al., 2012). Thus, it will be crucial to understand to what extent complement components and cytokines identified here contribute to the selective pressure that results in aging-induced clonality and increased incidence of hematologic neoplasia (Henry et al., 2015; Cooper and Young, 2017). In aged CARc and SECs, we found overexpression of prototypical genes downstream of IFN, as well as TLR4 signaling axes, which to a great extent resembled those induced by infection-mimicking agents.

Another remarkable hallmark of aged BM stroma, similarly recapitulated by infection-mimicking molecules, is the generalized reduction of ECM genes and, most strongly, of different collagens, which has been observed in fibroblasts of other organs (Salzer et al., 2018). Matrisomic properties of the surrounding microenvironment are known to exert major influences in cell fate decisions in BM mimicking 3D cultures (Choi and Harley, 2017). However, teasing apart the specific biomechanical and molecular effects of these structures in vivo has been challenging so far due to the daunting complexity of matrix fiber scaffolds. Our data suggest that ECM composition is (1) largely derived from mesenchyme but molecularly compartmentalized between cellular subsets, and (2) very dynamically and specifically shaped not only in aging, but also throughout postnatal development and as part of rapid stereotypic responses to inflammatory challenge. It is therefore tempting to speculate that matrisomic scaffolds represent a malleable and actionable platform for the swift modulation of hematopoiesis by stromal cells in different pathophysiological contexts.

In summary, the temporally resolved, population-based, transcriptomic profiles of stromal subsets presented here represent powerful resources for the dissection of unappreciated functional aspects of stromal cells and the understanding of changes in stromal-hematopoietic crosstalk that lead to modulation of BM function throughout the lifespan of an organism.

\section{STAR $\star$ METHODS}

Detailed methods are provided in the online version of this paper and include the following:

- KEY RESOURCES TABLE

- LEAD CONTACT AND MATERIALS AVAILABILITY

- EXPERIMENTAL MODEL AND SUBJECT DETAILS

O Mice

O Experimental conditions

- METHOD DETAILS

O Stromal cell isolation

O Cell sorting and flow cytometry analysis

O RNA isolation, library preparation, cluster generation and sequencing

Immunostaining and volumetric confocal imaging

O Bioinformatic analyses

- QUANTIFICATION AND STATISTICAL ANALYSIS

- DATA AND CODE AVAILABILITY

\section{SUPPLEMENTAL INFORMATION}

Supplemental Information can be found online at https://doi.org/10.1016/j celrep.2019.11.004.

\section{ACKNOWLEDGMENTS}

We thank Daniel Pinschewer (University of Zurich) for providing the I/33-Gfp knock-in mice and Takashi Nagasawa for the Cxc/12-GFP mouse strain P.M.H. is supported by fellowship from the Forschungskredit of the University of Zurich. This work was supported by the Swiss National Science Foundation (310030B_166673/1 to M.G.M. and 31003A_159597/1), Swiss Cancer League (KFS-3986-08-2016), FP7 Marie Curie Career Integration Grant (PCIG13-GA2013-618633) from the European Union, and the Helmut Horten Foundation (Lugano, Switzerland) to C.N-A. The Bioinformatics Unit is supported by the Instituto de Salud Carlos III (ISCIII), Spanish National Bioinformatics Institute (INB) (PT17/0009/0011-ISCIII-SGEFI/ERDF), Project RETOS RTI2018-097596-B-I00 (AEI-MICIU/ FEDER, UE) AEI-MCIU, and cofounded by the European Regional Development Fund (ERDF-EU). 


\section{AUTHOR CONTRIBUTIONS}

P.M.H. designed and performed experiments, analyzed data, and wrote the manuscript. R.G. designed and performed experiments. S.B. participated in the design of the study. E.P.-Y. and F.A.-S. designed the bioinformatic pipelines and performed data analysis. M.G.M. and C.N.-A. contributed equally, designed and directed the study, and wrote the manuscript.

\section{DECLARATION OF INTERESTS}

The authors declare no competing interests.

Received: July 15, 2019

Revised: October 3, 2019

Accepted: October 31, 2019

Published: December 3, 2019

\section{REFERENCES}

Acar, M., Kocherlakota, K.S., Murphy, M.M., Peyer, J.G., Oguro, H., Inra, C.N., Jaiyeola, C., Zhao, Z., Luby-Phelps, K., and Morrison, S.J. (2015). Deep imaging of bone marrow shows non-dividing stem cells are mainly perisinusoidal. Nature 526, 126-130.

Anders, S., Pyl, P.T., and Huber, W. (2015). HTSeq-a Python framework to work with high-throughput sequencing data. Bioinformatics 31, 166-169.

Andrews, S. (2010). FastQC: a quality control tool for high throughput sequence data (Babraham Institute).

Ara, T., Tokoyoda, K., Sugiyama, T., Egawa, T., Kawabata, K., and Nagasawa, T. (2003). Long-term hematopoietic stem cells require stromal cell-derived factor-1 for colonizing bone marrow during ontogeny. Immunity 19, 257-267.

Arora, N., Wenzel, P.L., McKinney-Freeman, S.L., Ross, S.J., Kim, P.G., Chou, S.S., Yoshimoto, M., Yoder, M.C., and Daley, G.Q. (2014). Effect of developmental stage of HSC and recipient on transplant outcomes. Dev. Cell 29, 621-628.

Baccin, C., Al-Sabah, J., Velten, L., Helbling, P.M., Grünschläger, F., Hernández-Malmierca, P., Nombela Arrieta, C., Steinmetz, L.M., Trumpp, A., and Haas, S. (2019). Combined single-cell and spatial transcriptomics reveals the molecular, cellular and spatial bone marrow niche organization. bioRxiv. https://doi.org/10.1101/718395.

Baryawno, N., Przybylski, D., Kowalczyk, M.S., Kfoury, Y., Severe, N., Gustafsson, K., Kokkaliaris, K.D., Mercier, F., Tabaka, M., Hofree, M., et al. (2019). A Cellular Taxonomy of the Bone Marrow Stroma in Homeostasis and Leukemia. Cell 177, 1915-1932.

Beerman, I., Maloney, W.J., Weissmann, I.L., and Rossi, D.J. (2010). Stem cells and the aging hematopoietic system. Curr. Opin. Immunol. 22, 500-506. Benz, C., Copley, M.R., Kent, D.G., Wohrer, S., Cortes, A., Aghaeepour, N., Ma, E., Mader, H., Rowe, K., Day, C., et al. (2012). Hematopoietic stem cell subtypes expand differentially during development and display distinct lymphopoietic programs. Cell Stem Cell 10, 273-283.

Berg, J.S., Lin, K.K., Sonnet, C., Boles, N.C., Weksberg, D.C., Nguyen, H., Holt, L.J., Rickwood, D., Daly, R.J., and Goodell, M.A. (2011). Imprinted genes that regulate early mammalian growth are coexpressed in somatic stem cells. PLoS ONE 6, e26410.

Bernitz, J.M., Kim, H.S., MacArthur, B., Sieburg, H., and Moore, K. (2016). Hematopoietic stem cells count and remember self-renewal divisions. Cell 167, 1296-1309.

Bixel, M.G., Kusumbe, A.P., Ramasamy, S.K., Sivaraj, K.K., Butz, S., Vestweber, D., and Adams, R.H. (2017). Flow Dynamics and HSPC Homing in Bone Marrow Microvessels. Cell Rep. 18, 1804-1816.

Boeuf, S., Kunz, P., Hennig, T., Lehner, B., Hogendoorn, P., Bovée, J., and Richter, W. (2008). A chondrogenic gene expression signature in mesenchymal stem cells is a classifier of conventional central chondrosarcoma. J. Pathol. 216, 158-166.
Bowie, M.B., McKnight, K.D., Kent, D.G., McCaffrey, L., Hoodless, P.A., and Eaves, C.J. (2006). Hematopoietic stem cells proliferate until after birth and show a reversible phase-specific engraftment defect. J. Clin. Invest. 116, 2808-2816.

Butler, J.M., Nolan, D.J., Vertes, E.L., Varnum-Finney, B., Kobayashi, H., Hooper, A.T., Seandel, M., Shido, K., White, I.A., Kobayashi, M., et al. (2010). Endothelial cells are essential for the self-renewal and repopulation of Notch-dependent hematopoietic stem cells. Cell Stem Cell 6, 251-264.

Casanova-Acebes, M., Pitaval, C., Weiss, L.A., Nombela-Arrieta, C., Chèvre, R., A-González, N., Kunisaki, Y., Zhang, D., van Rooijen, N., Silberstein, L.E., et al. (2013). Rhythmic modulation of the hematopoietic niche through neutrophil clearance. Cell 153, 1025-1035.

Chambers, S.M., Boles, N.C., Lin, K.-Y.K., Tierney, M.P., Bowman, T.V., Bradfute, S.B., Chen, A.J., Merchant, A.A., Sirin, O., Weksberg, D.C., et al. (2007a). Hematopoietic fingerprints: an expression database of stem cells and their progeny. Cell Stem Cell 1, 578-591.

Chambers, S.M., Shaw, C.A., Gatza, C., Fisk, C.J., Donehower, L.A., and Goodell, M.A. (2007b). Aging hematopoietic stem cells decline in function and exhibit epigenetic dysregulation. PLoS Biol. 5, e201-e213.

Chavakis, T., Mitroulis, I., and Hajishengallis, G. (2019). Hematopoietic progenitor cells as integrative hubs for adaptation to and fine-tuning of inflammation. Nat. Immunol. 20, 802-811.

Choi, J.S., and Harley, B.A.C. (2017). Marrow-inspired matrix cues rapidly affect early fate decisions of hematopoietic stem and progenitor cells. Sci. Adv. 3, e1600455.

Chou, S., and Lodish, H.F. (2010). Fetal liver hepatic progenitors are supportive stromal cells for hematopoietic stem cells. Proc. Natl. Acad. Sci. USA 107, 7799-7804.

Chow, A., Lucas, D., Hidalgo, A., Méndez-Ferrer, S., Hashimoto, D., Scheiermann, C., Battista, M., Leboeuf, M., Prophete, C., van Rooijen, N., et al. (2011). Bone marrow CD169+ macrophages promote the retention of hematopoietic stem and progenitor cells in the mesenchymal stem cell niche. J. Exp. Med. 208, 261-271.

Cooper, J.N., and Young, N.S. (2017). Clonality in context: hematopoietic clones in their marrow environment. Blood 130, 2363-2372.

Corada, M., Orsenigo, F., Morini, M.F., Pitulescu, M.E., Bhat, G., Nyqvist, D., Breviario, F., Conti, V., Briot, A., Iruela-Arispe, M.L., et al. (2013). Sox17 is indispensable for acquisition and maintenance of arterial identity. Nat. Commun. 4, 2609 .

Cordeiro Gomes, A., Hara, T., Lim, V.Y., Herndler-Brandstetter, D., Nevius, E., Sugiyama, T., Tani-Ichi, S., Schlenner, S., Richie, E., Rodewald, H.-R., et al. (2016). Hematopoietic Stem Cell Niches Produce Lineage-Instructive Signals to Control Multipotent Progenitor Differentiation. Immunity 45, 1219-1231.

Dahlgren, M.W., Jones, S.W., Cautivo, K.M., Dubinin, A., Ortiz-Carpena, J.F., Farhat, S., Yu, K.S., Lee, K., Wang, C., Molofsky, A.V., et al. (2019). Adventitia Stromal Cells Define Group 2 Innate Lymphoid Cell Tissue Niches. Immunity $50,707-722$

de Haan, G., and Lazare, S.S. (2018). Aging of hematopoietic stem cells. Blood 131, 479-487.

de Magalhães, J.P., Curado, J., and Church, G.M. (2009). Meta-analysis of age-related gene expression profiles identifies common signatures of aging. Bioinformatics 25, 875-881.

Ding, L., Saunders, T.L., Enikolopov, G., and Morrison, S.J. (2012). Endothelial and perivascular cells maintain haematopoietic stem cells. Nature 481 , 457-462.

Eaves, C.J. (2015). Hematopoietic stem cells: concepts, definitions, and the new reality. Blood 125, 2605-2613.

Ernst, J., and Bar-Joseph, Z. (2006). STEM: a tool for the analysis of short time series gene expression data. BMC Bioinformatics 7, 191.

Espin-Palazon, R., Weijts, B., Mulero, V., and Traver, D. (2018). Proinflammatory Signals as Fuel for the Fire of Hematopoietic Stem Cell Emergence. Trends Cell Biol. 28, 58-66. 
Essers, M.A.G., Offner, S., Blanco-Bose, W.E., Waibler, Z., Kalinke, U., Duchosal, M.A., and Trumpp, A. (2009). IFNalpha activates dormant haematopoietic stem cells in vivo. Nature 458, 904-908.

Gazit, R., Garrison, B.S., Rao, T.N., Shay, T., Costello, J., Ericson, J., Kim, F., Collins, J.J., Regev, A., Wagers, A.J., and Rossi, D.J.; Immunological Genome Project Consortium (2013). Transcriptome analysis identifies regulators of hematopoietic stem and progenitor cells. Stem Cell Reports 1, 266-280.

Geiger, H., de Haan, G., and Florian, M.C. (2013). The ageing haematopoietic stem cell compartment. Nat. Rev. Immunol. 13, 376-389.

Gomariz, A., Helbling, P.M., Isringhausen, S., Suessbier, U., Becker, A., Boss, A., Nagasawa, T., Paul, G., Goksel, O., Székely, G., et al. (2018). Quantitative spatial analysis of haematopoiesis-regulating stromal cells in the bone marrow microenvironment by 3D microscopy. Nat. Commun. 9, 2532.

Graña, O., Rubio-Camarillo, M., Fdez-Riverola, F., Pisano, D.G., and GlezPeña, D. (2018). Nextpresso: Next generation sequencing expression analysis pipeline. Curr. Bioinform. 13, 583-591.

Greenbaum, A., Hsu, Y.-M.S., Day, R.B., Schuettpelz, L.G., Christopher, M.J., Borgerding, J.N., Nagasawa, T., and Link, D.C. (2013). CXCL12 in early mesenchymal progenitors is required for haematopoietic stem-cell maintenance. Nature 495, 227-230.

He, Q., Zhang, C., Wang, L., Zhang, P., Ma, D., Lv, J., and Liu, F. (2015). Inflammatory signaling regulates hematopoietic stem and progenitor cell emergence in vertebrates. Blood 125, 1098-1106.

Henry, C.J., Casás-Selves, M., Kim, J., Zaberezhnyy, V., Aghili, L., Daniel, A.E., Jimenez, L., Azam, T., McNamee, E.N., Clambey, E.T., et al. (2015). Agingassociated inflammation promotes selection for adaptive oncogenic events in B cell progenitors. J. Clin. Invest. 125, 4666-4680.

Himburg, H.A., Termini, C.M., Schlussel, L., Kan, J., Li, M., Zhao, L., Fang, T., Sasine, J.P., Chang, V.Y., and Chute, J.P. (2018). Distinct Bone Marrow Sources of Pleiotrophin Control Hematopoietic Stem Cell Maintenance and Regeneration. Cell Stem Cell 23, 370-381.

Hu, X., Garcia, M., Weng, L., Jung, X., Murakami, J.L., Kumar, B., Warden, C.D., Todorov, I., and Chen, C.-C. (2016). Identification of a common mesenchymal stromal progenitor for the adult haematopoietic niche. Nat. Commun. 7, 13095.

Itkin, T., Gur-Cohen, S., Spencer, J.A., Schajnovitz, A., Ramasamy, S.K., Kusumbe, A.P., Ledergor, G., Jung, Y., Milo, I., Poulos, M.G., et al. (2016). Distinct bone marrow blood vessels differentially regulate haematopoiesis. Nature 532, 323-328.

Kfoury, Y., and Scadden, D.T. (2015). Mesenchymal cell contributions to the stem cell niche. Cell Stem Cell 16, 239-253.

Ki, S., Park, D., Selden, H.J., Seita, J., Chung, H., Kim, J., Iyer, V.R., and Ehrlich, L.I.R. (2014). Global transcriptional profiling reveals distinct functions of thymic stromal subsets and age-related changes during thymic involution. Cell Rep. 9, 402-415.

Kim, I., Saunders, T.L., and Morrison, S.J. (2007). Sox17 dependence distinguishes the transcriptional regulation of fetal from adult hematopoietic stem cells. Cell 130, 470-483.

Kokkaliaris, K.D., Drew, E., Endele, M., Loeffler, D., Hoppe, P.S., Hilsenbeck, O., Schauberger, B., Hinzen, C., Skylaki, S., Theodorou, M., et al. (2016). Identification of factors promoting ex vivo maintenance of mouse hematopoietic stem cells by long-term single-cell quantification. Blood 128, 1181-1192.

Kovtonyuk, L.V., Fritsch, K., Feng, X., Manz, M.G., and Takizawa, H. (2016). Inflamm-Aging of Hematopoiesis, Hematopoietic Stem Cells, and the Bone Marrow Microenvironment. Front. Immunol. 7, 502.

Kusumbe, A.P., Ramasamy, S.K., and Adams, R.H. (2014). Coupling of angiogenesis and osteogenesis by a specific vessel subtype in bone. Nature 507, 323-328.

Kusumbe, A.P., Ramasamy, S.K., Itkin, T., Mäe, M.A., Langen, U.H., Betsholtz, C., Lapidot, T., and Adams, R.H. (2016). Age-dependent modulation of vascular niches for haematopoietic stem cells. Nature 532, 380-384.
Langmead, B., Trapnell, C., Pop, M., and Salzberg, S.L. (2009). Ultrafast and memory-efficient alignment of short DNA sequences to the human genome. Genome Biol. 10, R25.

Lazzari, E., and Butler, J.M. (2018). The Instructive Role of the Bone Marrow Niche in Aging and Leukemia. Curr. Stem Cell Rep. 4, 291-298.

Li, H. (2011). A statistical framework for SNP calling, mutation discovery, association mapping and population genetical parameter estimation from sequencing data. Bioinformatics 27, 2987-2993.

Li, X.M., Hu, Z., Jorgenson, M.L., and Slayton, W.B. (2009). High levels of acetylated low-density lipoprotein uptake and low tyrosine kinase with immunoglobulin and epidermal growth factor homology domains-2 (Tie2) promoter activity distinguish sinusoids from other vessel types in murine bone marrow. Circulation 120, 1910-1918.

Louis, I., Heinonen, K.M., Chagraoui, J., Vainio, S., Sauvageau, G., and Perreault, C. (2008). The signaling protein Wnt4 enhances thymopoiesis and expands multipotent hematopoietic progenitors through $\beta$-catenin-independent signaling. Immunity 29, 57-67.

Love, M.I., Huber, W., and Anders, S. (2014). Moderated estimation of fold change and dispersion for RNA-seq data with DESeq2. Genome Biol. 15, 550 Mahlakõiv, T., Flamar, A.L., Johnston, L.K., Moriyama, S., Putzel, G.G., Bryce, P.J., and Artis, D. (2019). Stromal cells maintain immune cell homeostasis in adipose tissue via production of interleukin-33. Sci. Immunol. 4, eaax0416.

Manz, M.G., and Boettcher, S. (2014). Emergency granulopoiesis. Nat. Rev. Immunol. 14, 302-314.

Martin, M. (2011). Cutadapt removes adapter sequences from highthroughput sequencing reads. EMBnet.journal 17, 10-12.

Maryanovich, M., Zahalka, A.H., Pierce, H., Pinho, S., Nakahara, F., Asada, N., Wei, Q., Wang, X., Ciero, P., Xu, J., et al. (2018). Adrenergic nerve degeneration in bone marrow drives aging of the hematopoietic stem cell niche. Nat. Med. 24, 782-791.

Mascarenhas, M.I., Parker, A., Dzierzak, E., and Ottersbach, K. (2009). Identification of novel regulators of hematopoietic stem cell development through refinement of stem cell localization and expression profiling. Blood 114, 4645-4653.

McGeer, E.G., Klegeris, A., and McGeer, P.L. (2005). Inflammation, the complement system and the diseases of aging. Neurobiol. Aging 26 (Supp/ 1), 94-97.

Mercier, F.E., Ragu, C., and Scadden, D.T. (2011). The bone marrow at the crossroads of blood and immunity. Nat. Rev. Immunol. 12, 49-60.

Mi, H., Muruganujan, A., Ebert, D., Huang, X., and Thomas, P.D. (2019). PANTHER version 14: more genomes, a new PANTHER GO-slim and improvements in enrichment analysis tools. Nucleic Acids Res. 47 (D1), D419-D426.

Mootha, V.K., Lindgren, C.M., Eriksson, K.-F., Subramanian, A., Sihag, S., Lehar, J., Puigserver, P., Carlsson, E., Ridderstråle, M., Laurila, E., et al. (2003) PGC-1alpha-responsive genes involved in oxidative phosphorylation are coordinately downregulated in human diabetes. Nat. Genet. 34, 267-273.

Morikawa, S., Mabuchi, Y., Kubota, Y., Nagai, Y., Niibe, K., Hiratsu, E., Suzuki, S., Miyauchi-Hara, C., Nagoshi, N., Sunabori, T., et al. (2009). Prospective identification, isolation, and systemic transplantation of multipotent mesenchymal stem cells in murine bone marrow. J. Exp. Med. 206, 2483-2496.

Moscatello, K.M., Biber, K.L., Dempsey, D.C., Chervenak, R., and Wolcott, R.M. (1998). Characterization of a B cell progenitor present in neonatal bone marrow and spleen but not in adult bone marrow and spleen. J. Immunol. 161, 5391-5398.

Naito, A.T., Sumida, T., Nomura, S., Liu, M.-L., Higo, T., Nakagawa, A., Okada, K., Sakai, T., Hashimoto, A., Hara, Y., et al. (2012). Complement C1q activates canonical Wnt signaling and promotes aging-related phenotypes. Cell 149 $1298-1313$

Nakamura-Ishizu, A., Takizawa, H., and Suda, T. (2014). The analysis, roles and regulation of quiescence in hematopoietic stem cells. Development 141 4656-4666. 
Nombela-Arrieta, C., and Isringhausen, S. (2017). The Role of the Bone Marrow Stromal Compartment in the Hematopoietic Response to Microbial Infections. Front. Immunol. 7, 689.

Nombela-Arrieta, C., and Manz, M.G. (2017). Quantification and three-dimensional microanatomical organization of the bone marrow. Blood Adv. 1, 407-416.

Nusspaumer, G., Jaiswal, S., Barbero, A., Reinhardt, R., Ishay Ronen, D., Haumer, A., Lufkin, T., Martin, I., and Zeller, R. (2017). Ontogenic Identification and Analysis of Mesenchymal Stromal Cell Populations during Mouse Limb and Long Bone Development. Stem Cell Reports 9, 1124-1138.

Oboki, K., Ohno, T., Kajiwara, N., Arae, K., Morita, H., Ishii, A., Nambu, A., Abe, T., Kiyonari, H., Matsumoto, K., et al. (2010). IL-33 is a crucial amplifier of innate rather than acquired immunity. Proc. Natl. Acad. Sci. USA 107, 18581-18586.

Omatsu, Y., Sugiyama, T., Kohara, H., Kondoh, G., Fujii, N., Kohno, K., and Nagasawa, T. (2010). The essential functions of adipo-osteogenic progenitors as the hematopoietic stem and progenitor cell niche. Immunity 33, 387-399.

Omatsu, Y., Seike, M., Sugiyama, T., Kume, T., and Nagasawa, T. (2014). Foxc1 is a critical regulator of haematopoietic stem/progenitor cell niche formation. Nature 508, 536-540.

Park, M.H., Jin, H.K., Min, W.K., Lee, W.W., Lee, J.E., Akiyama, H., Herzog, H., Enikolopov, G.N., Schuchman, E.H., and Bae, J.S. (2015). Neuropeptide Y regulates the hematopoietic stem cell microenvironment and prevents nerve injury in the bone marrow. EMBO J. 34, 1648-1660.

Pietras, E.M., Mirantes-Barbeito, C., Fong, S., Loeffler, D., Kovtonyuk, L.V., Zhang, S., Lakshminarasimhan, R., Chin, C.P., Techner, J.-M., Will, B., et al. (2016). Chronic interleukin-1 exposure drives haematopoietic stem cells towards precocious myeloid differentiation at the expense of self-renewal. Nat. Cell Biol. 18, 607-618.

Pihlgren, M., Schallert, N., Tougne, C., Bozzotti, P., Kovarik, J., Fulurija, A., Kosco-Vilbois, M., Lambert, P.H., and Siegrist, C.A. (2001). Delayed and deficient establishment of the long-term bone marrow plasma cell pool during early life. Eur. J. Immunol. 31, 939-946.

Ratajczak, M.Z., Kim, C.H., Wojakowski, W., Janowska-Wieczorek, A., Kucia, M., and Ratajczak, J. (2010). Innate immunity as orchestrator of stem cell mobilization. Leukemia 24, 1667-1675.

Salzer, M.C., Lafzi, A., Berenguer-Llergo, A., Youssif, C., Castellanos, A., Solanas, G., Peixoto, F.O., Stephan-Otto Attolini, C., Prats, N., Aguilera, M., et al. (2018). Identity Noise and Adipogenic Traits Characterize Dermal Fibroblast Aging. Cell 175, 1575-1590.

Sawamiphak, S., Kontarakis, Z., and Stainier, D.Y.R. (2014). Interferon gamma signaling positively regulates hematopoietic stem cell emergence. Dev. Cell 31, 640-653.

Schreck, C., Istvánffy, R., Ziegenhain, C., Sippenauer, T., Ruf, F., Henkel, L., Gärtner, F., Vieth, B., Florian, M.C., Mende, N., et al. (2017). Niche WNT5A regulates the actin cytoskeleton during regeneration of hematopoietic stem cells. J. Exp. Med. 214, 165-181.

Schumacher, A., Denecke, B., Braunschweig, T., Stahlschmidt, J., Ziegler, S., Brandenburg, L.-O., Stope, M.B., Martincuks, A., Vogt, M., Görtz, D., et al. (2015). Angptl4 is upregulated under inflammatory conditions in the bone marrow of mice, expands myeloid progenitors, and accelerates reconstitution of platelets after myelosuppressive therapy. J. Hematol. Oncol. 8, 64.

Schürch, C.M., Riether, C., and Ochsenbein, A.F. (2014). Cytotoxic CD8+ $\mathrm{T}$ cells stimulate hematopoietic progenitors by promoting cytokine release from bone marrow mesenchymal stromal cells. Cell Stem Cell 14, 460-472.

Shi, C., Jia, T., Méndez-Ferrer, S., Hohl, T.M., Serbina, N.V., Lipuma, L., Leiner, I., Li, M.O., Frenette, P.S., and Pamer, E.G. (2011). Bone marrow mesenchymal stem and progenitor cells induce monocyte emigration in response to circulating toll-like receptor ligands. Immunity 34, 590-601.

Singh, P., Hoggatt, J., Kamocka, M.M., Mohammad, K.S., Saunders, M.R., Li, H., Speth, J., Carlesso, N., Guise, T.A., and Pelus, L.M. (2017). Neuropeptide Y regulates a vascular gateway for hematopoietic stem and progenitor cells. J. Clin. Invest. 127, 4527-4540.
Sitnik, K.M., Wendland, K., Weishaupt, H., Uronen-Hansson, H., White, A.J., Anderson, G., Kotarsky, K., and Agace, W.W. (2016). Context-Dependent Development of Lymphoid Stroma from Adult CD34(+) Adventitial Progenitors. Cell Rep. 14, 2375-2388.

Sivaraj, K.K., and Adams, R.H. (2016). Blood vessel formation and function in bone. Development 143, 2706-2715.

Smith-Berdan, S., Nguyen, A., Hong, M.A., and Forsberg, E.C. (2015). ROBO4mediated vascular integrity regulates the directionality of hematopoietic stem cell trafficking. Stem Cell Reports 4, 255-268.

Spallanzani, R.G., Zemmour, D., Xiao, T., Jayewickreme, T., Li, C., Bryce, P.J., Benoist, C., and Mathis, D. (2019). Distinct immunocyte-promoting and adipocyte-generating stromal components coordinate adipose tissue immune and metabolic tenors. Sci. Immunol. 4, eaaw3658.

Stegner, D., vanEeuwijk, J.M.M., Angay, O., Gorelashvili, M.G., Semeniak, D., Pinnecker, J., Schmithausen, P., Meyer, I., Friedrich, M., Dütting, S., et al. (2017). Thrombopoiesis is spatially regulated by the bone marrow vasculature. Nat. Commun. 8, 127.

Subramanian, A., Tamayo, P., Mootha, V.K., Mukherjee, S., Ebert, B.L., Gillette, M.A., Paulovich, A., Pomeroy, S.L., Golub, T.R., Lander, E.S., and Mesirov, J.P. (2005). Gene set enrichment analysis: a knowledge-based approach for interpreting genome-wide expression profiles. Proc. Natl. Acad. Sci. USA 102, 15545-15550.

Sugiyama, T., Kohara, H., Noda, M., and Nagasawa, T. (2006). Maintenance of the hematopoietic stem cell pool by CXCL12-CXCR4 chemokine signaling in bone marrow stromal cell niches. Immunity 25, 977-988.

Sun, D., Luo, M., Jeong, M., Rodriguez, B., Xia, Z., Hannah, R., Wang, H., Le, T., Faull, K.F., Chen, R., et al. (2014). Epigenomic profiling of young and aged HSCs reveals concerted changes during aging that reinforce self-renewal. Cell Stem Cell 14, 673-688.

Takizawa, H., Regoes, R.R., Boddupalli, C.S., Bonhoeffer, S., and Manz, M.G. (2011). Dynamic variation in cycling of hematopoietic stem cells in steady state and inflammation. J. Exp. Med. 208, 273-284.

Takizawa, H., Boettcher, S., and Manz, M.G. (2012). Demand-adapted regulation of early hematopoiesis in infection and inflammation. Blood 119, $2991-$ 3002.

Takizawa, H., Fritsch, K., Kovtonyuk, L.V., Saito, Y., Yakkala, C., Jacobs, K., Ahuja, A.K., Lopes, M., Hausmann, A., Hardt, W.-D., et al. (2017). PathogenInduced TLR4-TRIF Innate Immune Signaling in Hematopoietic Stem Cells Promotes Proliferation but Reduces Competitive Fitness. Cell Stem Cell 21 225-240.

Tanegashima, K., Suzuki, K., Nakayama, Y., Tsuji, K., Shigenaga, A., Otaka, A., and Hara, T. (2013). CXCL14 is a natural inhibitor of the CXCL12-CXCR4 signaling axis. FEBS Lett. 587, 1731-1735.

Thomas, D.D., Sommer, A.G., Balazs, A.B., Beerman, I., Murphy, G.J., Rossi, D., and Mostoslavsky, G. (2016). Insulin-like growth factor 2 modulates murine hematopoietic stem cell maintenance through upregulation of p57. Exp. Hematol. 44, 422-433.

Tikhonova, A.N., Dolgalev, I., Hu, H., Sivaraj, K.K., Hoxha, E., Cuesta-Domínguez, Á., Pinho, S., Akhmetzyanova, I., Gao, J., Witkowski, M., et al. (2019). The bone marrow microenvironment at single-cell resolution. Nature 569, 222-228.

Tokoyoda, K., Hauser, A.E., Nakayama, T., and Radbruch, A. (2010). Organization of immunological memory by bone marrow stroma. Nat. Rev. Immunol. 10, 193-200.

Trapnell, C., Roberts, A., Goff, L., Pertea, G., Kim, D., Kelley, D.R., Pimentel, H., Salzberg, S.L., Rinn, J.L., and Pachter, L. (2012). Differential gene and transcript expression analysis of RNA-seq experiments with TopHat and Cufflinks. Nat. Protoc. 7, 562-578.

Trompouki, E. (2016). Stress and Non-Stress Roles of Inflammatory Signals during HSC Emergence and Maintenance. Front Immunol. 7, 487.

Ueda, Y., Yang, K., Foster, S.J., Kondo, M., and Kelsoe, G. (2004). Inflammation controls $B$ lymphopoiesis by regulating chemokine CXCL12 expression. J. Exp. Med. 199, 47-58. 
Ueda, Y., Kondo, M., and Kelsoe, G. (2005). Inflammation and the reciprocal production of granulocytes and lymphocytes in bone marrow. J. Exp. Med. 201, 1771-1780.

Venkatraman, A., He, X.C., Thorvaldsen, J.L., Sugimura, R., Perry, J.M., Tao, F., Zhao, M., Christenson, M.K., Sanchez, R., Yu, J.Y., et al. (2013). Maternal imprinting at the H19-Igf2 locus maintains adult haematopoietic stem cell quiescence. Nature 500, 345-349.

Walter, D., Lier, A., Geiselhart, A., Thalheimer, F.B., Huntscha, S., Sobotta, M.C., Moehrle, B., Brocks, D., Bayindir, I., Kaschutnig, P., et al. (2015). Exit from dormancy provokes DNA-damage-induced attrition in haematopoietic stem cells. Nature 520, 549-552.

Welner, R.S., Amabile, G., Bararia, D., Czibere, A., Yang, H., Zhang, H., Pontes, L.L., Ye, M., Levantini, E., Di Ruscio, A., et al. (2015). Treatment of chronic myelogenous leukemia by blocking cytokine alterations found in normal stem and progenitor cells. Cancer Cell 27, 671-681.

Wilson, A., Laurenti, E., Oser, G., van der Wath, R.C., Blanco-Bose, W., Jaworski, M., Offner, S., Dunant, C.F., Eshkind, L., Bockamp, E., et al. (2008). Hematopoietic stem cells reversibly switch from dormancy to self-renewal during homeostasis and repair. Cell 135, 1118-1129.

Wingett, S.W., and Andrews, S. (2018). FastQ Screen: A tool for multi-genome mapping and quality control. F1000Res. 7, 1338.

Wolock, S.L., Krishnan, I., Tenen, D.E., Matkins, V., Camacho, V., Patel, S., Agarwal, P., Bhatia, R., Tenen, D.G., Klein, A.M., and Welner, R.S. (2019). Mapping Distinct Bone Marrow Niche Populations and Their Differentiation Paths. Cell Rep. 28, 302-311.
Wu, Q., Kawahara, M., and Kono, T. (2008). Synergistic role of Igf2 and Dlk1 in fetal liver development and hematopoiesis in bi-maternal mice. J. Reprod. Dev. 54, 177-182.

Xiao, Y. (2015). Loss of Angiopoietin-like 7 diminishes the regeneration capacity of hematopoietic stem and progenitor cells 1-5. J. Hematol. Oncol. 8, 7.

Xiao, Y., Jiang, Z., Li, Y., Ye, W., Jia, B., Zhang, M., Xu, Y., Wu, D., Lai, L., Chen, Y., et al. (2015). ANGPTL7 regulates the expansion and repopulation of human hematopoietic stem and progenitor cells. Haematologica 100, 585-594.

Xu, C., Gao, X., Wei, Q., Nakahara, F., Zimmerman, S.E., Mar, J., and Frenette, P.S. (2018). Stem cell factor is selectively secreted by arterial endothelial cells in bone marrow. Nat. Commun. 9, 2449.

Ye, M., Zhang, H., Amabile, G., Yang, H., Staber, P.B., Zhang, P., Levantini, E., Alberich-Jordà, M., Zhang, J., Kawasaki, A., and Tenen, D.G. (2013). C/EBPa controls acquisition and maintenance of adult haematopoietic stem cell quiescence. Nat. Cell Biol. 15, 385-394.

Zhang, H., Rodriguez, S., Wang, L., Wang, S., Serezani, H., Kapur, R., Cardoso, A.A., and Carlesso, N. (2016). Sepsis Induces Hematopoietic Stem Cell Exhaustion and Myelosuppression through Distinct Contributions of TRIF and MYD88. Stem Cell Reports 6, 940-956.

Zhou, B.O., Yue, R., Murphy, M.M., Peyer, J.G., and Morrison, S.J. (2014). Leptin-receptor-expressing mesenchymal stromal cells represent the main source of bone formed by adult bone marrow. Cell Stem Cell 15, 154-168. 


\section{STAR $\star$ METHODS}

\section{KEY RESOURCES TABLE}

\begin{tabular}{|c|c|c|}
\hline REAGENT or RESOURCE & SOURCE & IDENTIFIER \\
\hline \multicolumn{3}{|l|}{ Antibodies } \\
\hline Podoplanin (eBio8.1.1) & Thermo Fisher Scientific & Cat\# 14-5381-85; RRID: AB_1210507 \\
\hline F4/80 (BM8) & Thermo Fisher Scientific & Cat\# 14-4801-82; RRID: AB_467558 \\
\hline Collagen IV & Abcam & Cat\# ab6586; RRID: AB_305584 \\
\hline Endomucin (V.7C7) & Santa Cruz Biotechnology & Cat\# Sc-65495; RRID: AB_2100037 \\
\hline Ly-6A/E (Sca-1) & BioLegend & Cat\# 122502; RRID: AB_756187 \\
\hline CD34 (RAM34) & Thermo Fisher Scientific & Cat\# 14-0341-81; RRID: AB_467209 \\
\hline $\begin{array}{l}\text { Living Colors } ® \text { Full-Length GFP Polyclonal } \\
\text { Antibody }\end{array}$ & Takara Bio & Cat\# 632592; RRID: AB_2336883 \\
\hline Biotin Goat Anti-Syrian Hamster IgG & Jackson ImmunoResearch & Cat\# 107-065-142; RRID: AB_2337458 \\
\hline Donkey anti-Goat IgG $(\mathrm{H}+\mathrm{L})$, AF546 & Thermo Fisher Scientific & Cat\# A-11056; RRID: AB_2534103 \\
\hline Donkey anti-Goat IgG $(\mathrm{H}+\mathrm{L}), \mathrm{AF} 680$ & Thermo Fisher Scientific & Cat\# A-21084; RRID: AB_2535741 \\
\hline Donkey anti-Rat IgG (H+L), AF594 & Thermo Fisher Scientific & Cat\# A-21209; RRID: AB_2535795 \\
\hline Donkey anti-Rat IgG $(\mathrm{H}+\mathrm{L})$, AF488 & Thermo Fisher Scientific & Cat\# A-21208; RRID: AB_2535794 \\
\hline Donkey anti-Rabbit lgG (H+L), AF680 & Thermo Fisher Scientific & Cat\# A10043; RRID: AB_2534018 \\
\hline Donkey anti-Rabbit IgG $(\mathrm{H}+\mathrm{L})$, AF546 & Thermo Fisher Scientific & Cat\# A10040; RRID: AB_2534016 \\
\hline Streptavidin, Alexa Fluor 555 conjugate & Thermo Fisher Scientific & Cat\# S32355; RRID: AB_2571525 \\
\hline TruStain fcX (anti-mouse CD16/32) & BioLegend & Cat\# 101320; RRID: AB_1574975 \\
\hline CD45 (30-F11), PerCP-Cyanine5.5 & Thermo Fisher Scientific & Cat\# 45-0451-82; RRID: AB_1107002 \\
\hline TER-119, PerCP-Cyanine5.5 & Thermo Fisher Scientific & Cat\# 45-5921-82; RRID: AB_925765 \\
\hline CD31 (PECAM-1), PE-Cyanine7 & Thermo Fisher Scientific & Cat\# 25-0311-82; RRID: AB_2716949 \\
\hline CD140b (PDGFRB), APC & Thermo Fisher Scientific & Cat\# 17-1402-82 RRID: AB_1548743 \\
\hline CD105 (MJ7/18), BV786 & BD Biosciences & Cat\# 564746; RRID: AB_2732065 \\
\hline Ly-6A/E (Sca-1), APC/Cy7 & BioLegend & Cat\# 108126; RRID: AB_10645327 \\
\hline CD206 (MMR), BV711 & BioLegend & Cat\# 141727; RRID: AB_2565822 \\
\hline Rat IgG2a, $\kappa$ Isotype Control, BV711 & BioLegend & Cat\# 400551 \\
\hline Podoplanin (eBio8.1.1), PE & Thermo Fisher Scientific & Cat\# 12-5381-82; RRID: AB_1907439 \\
\hline Syrian Hamster IgG, Isotype Control, PE & Thermo Fisher Scientific & Cat\# 12-4914-81; RRID: AB_470075 \\
\hline CD34 (RAM34), PE & BD Biosciences & Cat\# 551387; RRID: AB_394176 \\
\hline \multicolumn{3}{|l|}{ Chemicals, Peptides, and Recombinant Proteins } \\
\hline Poly(I:C) (HMW) & InvivoGen & Cat\# t|r|-pic \\
\hline LPS-EB Ultrapure (LPS from E. coli O111:B4) & InvivoGen & Cat\# t|rl-3pelps \\
\hline Collagenase, Type 2 & Worthington Biochemical & Cat\# LS004176 \\
\hline Deoxyribonuclease I & Worthington Biochemical & Cat\# LS002007 \\
\hline RapiClear® 1.52 & SunJin Lab & Cat\# RC152001 \\
\hline EDTA solution pH $8.0(0.5 \mathrm{M})$ & Panreac Appli-Chem & Cat\# A4892,0100 \\
\hline DMEM, high glucose, GlutaMAX suppl. & Thermo Fisher Scientific & Cat\# 61965059 \\
\hline HEPES (1M) & Thermo Fisher Scientific & Cat\# 15630056 \\
\hline $16 \%$ Paraformaldehyde & EMS & Cat\# 15710 \\
\hline 2-Mercaptoethanol & Sigma & Cat\# M3148 \\
\hline \multicolumn{3}{|l|}{ Critical Commercial Assays } \\
\hline RNeasy Plus Micro Kit & QIAGEN & Cat\# 74034 \\
\hline $\begin{array}{l}\text { SMARTer } \circledast \text { Stranded Total RNA-Seq V1- Pico } \\
\text { Input Mammalian }\end{array}$ & Takara Bio & Cat\# 635007 \\
\hline
\end{tabular}




\begin{tabular}{|c|c|c|}
\hline Continued & & \\
\hline REAGENT or RESOURCE & SOURCE & IDENTIFIER \\
\hline \multicolumn{3}{|l|}{ Deposited Data } \\
\hline RNA sequencing data & This study & GEO accession: GSE133922 \\
\hline \multicolumn{3}{|l|}{ Experimental Models: Organisms/Strains } \\
\hline Mouse: C57BL/6JRj & Janvier Labs, France & $\mathrm{N} / \mathrm{A}$ \\
\hline Mouse: II33-GFP & RIKEN, Japan & MGl:4838250 \\
\hline Mouse: Cxcl12-GFP & Osaka, Japan & MGI:1934384 \\
\hline \multicolumn{3}{|l|}{ Software and Algorithms } \\
\hline FlowJo v10 & FlowJo, LLC & https://www.flowjo.com \\
\hline Imaris software (v8.41) & Oxford Instruments & https://imaris.oxinst.com/packages \\
\hline Nextpresso pipeline v.1.9.2 & Graña et al., 2018 & https://github.com/osvaldogc/nextpresso1.9.2 \\
\hline FastQC v0.11.3 & Andrews, 2010 & $N / A$ \\
\hline FastQ Screen v0.5.2 & Wingett and Andrews, 2018 & N/A \\
\hline Cutadapt & Martin, 2011 & N/A \\
\hline TopHat 2.0.10 & Trapnell et al., 2012 & N/A \\
\hline Bowtie 1.0.0 & Langmead et al., 2009 & $N / A$ \\
\hline SAMtools 0.1 .19 & $\mathrm{Li}, 2011$ & N/A \\
\hline HTSeq framework 0.6.1 & Anders et al., 2015 & N/A \\
\hline DESeq2 v1.18.1 & Love et al., 2014 & $N / A$ \\
\hline Cufflinks V2.2.1 & Trapnell et al., 2012 & $N / A$ \\
\hline $\mathrm{R}(\mathrm{v} 3.4 .1)$ & & https://www.r-project.org \\
\hline Short Time-series Expression Miner (STEM) & Ernst and Bar-Joseph, 2006 & N/A \\
\hline PANTHER & Mi et al., 2019 & N/A \\
\hline GSEA & $\begin{array}{l}\text { Mootha et al., 2003; } \\
\text { Subramanian et al., } 2005\end{array}$ & http://software.broadinstitute.org/gsea/index.jsp \\
\hline Heatmaps & Broad Institute & https://software.broadinstitute.org/morpheus \\
\hline \multicolumn{3}{|l|}{ Other } \\
\hline Nonstick, RNase-free Microfuge Tubes & Thermo Fisher Scientific & Cat\# AM12450 \\
\hline Dow Corning ${ }^{\circledR}$ high-vacuum silicone grease & Sigma & Cat\# Z273554 \\
\hline Transcript annotations Mus musculus & $\begin{array}{l}\text { https://ccb.jhu.edu/software/ } \\
\text { tophat/igenomes.shtml }\end{array}$ & $\mathrm{GRCm} 38 / \mathrm{mm} 10$ \\
\hline
\end{tabular}

\section{LEAD CONTACT AND MATERIALS AVAILABILITY}

Additional information and requests for materials, should be addressed to, and will be fulfilled by the lead contact of this study, César Nombela-Arrieta (Cesar.NombelaArrieta@usz.ch). This study did not generate new unique reagents.

\section{EXPERIMENTAL MODEL AND SUBJECT DETAILS}

Mice

C57BL/6JRj mice were purchased from Janvier Labs (France) and maintained under standard conditions at the animal facility of the University Hospital Zurich. I/33-GFP mice were generated by the RIKEN Center for Life Science Technologies (Kobe, Japan) (Accession number CDB0631K http://www2.clst.riken.jp/arg/mutant\%20mice\%20list.html) (Oboki et al., 2010). Cxcl12-GFP mice were kindly provided by Takashi Nagasawa (Ara et al., 2003). All the experimental procedures involving animal models were approved by the veterinarian office of the Canton of Zurich, Switzerland.

\section{Experimental conditions}

Only male animals were used for RNA-seq experiments. Mice were analyzed at different ages, namely 2 weeks (14-15 days old, termed juvenile), 2 months (8-9 weeks old, designated as adult), 2 year-old (20-24 months old, aged mice). LPS-challenged mice (8-9 weeks old) were injected twice intraperitoneally with $35 \mu \mathrm{g}$ of lipopolysaccharides (in $200 \mu \mathrm{L}$ phosphate buffered saline). pl:C-challenged mice (8-9 weeks old) were injected twice intraperitoneally with $100 \mu \mathrm{g}$ of polyinosinic-polycytidylic acid (in $200 \mu \mathrm{L}$ phosphate buffered saline). The injections were performed 48 hours and 4 hours before sacrifice. Four independent biological 
replicates, which were collected at different times and dates were processed and sequenced for each experimental group. All replicates, cell types and conditions were sequenced at once.

\section{METHOD DETAILS}

\section{Stromal cell isolation}

The isolation procedure for stromal cells has been previously described in detail in Gomariz et al. (2018). Briefly, two sets of femur/ tibia/pelvis were isolated from the surrounding tissue and cleaned using a surgical scalpel and paper tissues in order to thoroughly remove surrounding muscle and connective tissue. The BM content was flushed out into 6-well plates utilizing a $26 \mathrm{G}$ syringe and $5 \mathrm{~mL}$ of digestion medium (DMEM GlutaMAX, $10 \mathrm{mM} \mathrm{HEPES,} 10 \%$ fetal bovine serum). The remaining bone enclosures were carefully cut into small fragments (approximately $1 \mathrm{~mm}^{3}$ in size) using scissors and added to the same well already containing digestion medium and the previously flushed marrow. For the enzymatic tissue digestion, collagenase $(0.04 \mathrm{~g} / \mathrm{mL}) \mathrm{and} \mathrm{DNase}(0.2 \mathrm{mg} / \mathrm{mL})$ were added and thoroughly mixed using a $1 \mathrm{~mL}$ pipette and the cell suspension incubated for 45 minutes at $37^{\circ} \mathrm{C}$ with gentle sample agitation. Next, $5 \mathrm{~mL}$ of ice-cold calcium- and magnesium-free phosphate-buffered saline (PBS) containing 10\% FBS were added and the suspension filtered through a $70 \mu \mathrm{m}$ cell strainer and washed once more with PBS $\left(4^{\circ} \mathrm{C}\right)$.

\section{Cell sorting and flow cytometry analysis}

Cell suspensions were blocked using TruStain $\mathrm{fcX}^{\mathrm{TM}}$ for 15 mins at $4^{\circ} \mathrm{C}$ and subsequently immunostained using pre-conjugated surface antibodies for 30 minutes at $4^{\circ} \mathrm{C}$. All antibodies employed in this study, including commercial sources and concentrations are shown in the key resource table. After staining, the cells were washed twice using ice-cold PBS and resuspended in PBS containing DAPI $(0.5 \mu \mathrm{g} / \mathrm{mL})$ and analyzed using an LSR II Fortessa (BD Biosciences). Post-acquisition data analysis was done using FlowJo 10 software. Cell sorting was performed using a FACS Aria (BD Biosciences) and all four stromal cell populations were sorted directly into RNase-free microfuge tubes (ThermoFisher) containing RLT lysis buffer (QIAGEN) at 4 ${ }^{\circ} \mathrm{C}$ until 4'000 to $50^{\prime} 000$ cells were collected for each cell type. In order to collect enough cells, the cell extracts of two mice were combined for each replicate before cell sorting.

RNA isolation, library preparation, cluster generation and sequencing Following the manufacturer's instructions of the RNeasy Plus Micro Kit (QIAGEN), genomic DNA was depleted using gDNA eliminator columns and RNA extracted from the cell lysates. The quality of the isolated RNA was measured using a Bioanalyzer 2100 (Agilent, Waldbronn, Germany). Library preparation, cluster generation, and sequencing were performed at the functional genomics center Zurich (FGCZ, Zurich, Switzerland). The libraries were prepared using the SMARTer ${ }^{\circledR}$ Stranded Total RNA-Seq - Pico Input Mammalian - kit (Takara Bio, USA) following the manufacturer's instructions. For each sample 600 pg of input total RNA were used. The TruSeq SR Cluster Kit v4-cBot-HS (Illumina, Inc, California, USA) was employed for cluster generation using 8 pM of pooled normalized libraries on the cBOT. Sequencing was performed on the Illumina HiSeq 4000 single end 125 bp using the TruSeq SBS Kit v4-HS (Illumina, Inc, California, USA).

\section{Immunostaining and volumetric confocal imaging}

3D imaging protocols have been previously described in detail (Gomariz et al., 2018). Briefly, femurs were isolated and thoroughly cleaned from the surrounding tissue, fixed in $2 \%$ paraformaldehyde in PBS $\left(6 \mathrm{~h}, 4^{\circ} \mathrm{C}\right)$, dehydrated in $30 \%$ sucrose in PBS $(72 \mathrm{~h}$, $4^{\circ} \mathrm{C}$ ), embedded in OCT medium, snap-frozen and bi-laterally sectioned using a cryotome. The obtained thick BM slices were incubated in blocking solution overnight $\left(0.2 \%\right.$ Triton $\mathrm{X}-100,1 \%$ bovine serum albumin, $10 \%$ donkey serum, in PBS) at $4{ }^{\circ} \mathrm{C}$ and subsequently stained with primary and secondary antibodies diluted in blocking solution for 72 hours each, including a $3 \times 1$ hour washing step (PBS) in between. The samples were optically cleared in RapiClear 1.52 by immersion for 6 hours. Imaging was performed on a SP8 Leica confocal microscope. Imaris software (v8.41) was used for analysis and rendering of microscopy data.

\section{Bioinformatic analyses}

Processing of RNAseq data was done using the Nextpresso pipeline v.1.9.2 (Graña et al., 2018). The quality of the raw data was assessed using FastQC v0.11.3 (Andrews, 2010) and FastQ Screen v0.5.2 (Wingett and Andrews, 2018) tools. Cutadapt (Martin, 2011) was used to remove the adaptors, trim the first three bases of the reads and the $3^{\prime}$ and $5^{\prime}$ end nucleotides with a quality cutoff of 20 , requiring a minimum length of 40. Reads were then aligned with TopHat 2.0.10 (Trapnell et al., 2012) using Bowtie 1.0.0 (Langmead et al., 2009) and SAMtools 0.1 .19 (Li, 2011) against the GRCm38/mm10 assembly of the mouse genome containing only protein coding genes. During the alignment only 2 mismatches and 20 multihits were allowed. Transcripts were quantified using htseq-count from HTSeq framework 0.6.1 (Anders et al., 2015) and the differential expression test was done with DESeq2 v1.18.1 (Love et al., 2014) using the Mus musculus GRCm38/mm10 transcript annotations from https://ccb.jhu.edu/software/tophat/igenomes.shtml. Fragments Per Kilobase of exon per Million fragments mapped (FPKM) expression values were computed using the Cuffquant and Cuffnorm functions included in Cufflinks V2.2.1 suite (Trapnell et al., 2012). Only genes showing enough global expression and variability were kept ( $>2$ FPKM in $>20 \%$ of the samples, IQR range $>1$ ). 
To check for similarities between replicates a Pearson correlation test was applied over the FPKMs. Only one sample displayed a correlation coefficient smaller than 0.90 in a comparison to its replicates and was therefore excluded from the analysis.

Principal Component Analysis (PCA) and Hierarchical Clustering were created using R (v3.4.1) and the ggpubr and dendextend packages over the normalized FPKM expression values. The Hierarchical Clustering used the Euclidian distance and the Ward.D2 algorithm as the clustering method. In PCA plots, individual dots represent different biological replicates.

Gene set enrichments were calculated using the GSEA software (Broad Institute) using the previously defined gene sets from the Molecular Signatures Database (MSigDB 6.2) (Mootha et al., 2003; Subramanian et al., 2005). The gene list was ranked according to $\log _{2}$ FCs from the differential expression test and the pre-ranked GSEA was run using the classic enrichment statistic with 10 '000 permutations.

Gene ontology analysis was performed using the PANTHER Overrepresentation Test (GO database release: 2018-04-04). For each test a background list containing all detected genes in this study was provided and statistics evaluated using Fisher's Exact test with FDR multiple test correction (Mi et al., 2019). Additionally, larger GO categories were selected by excluding GO terms that contained less than 10 genes in the background list.

The Short Time-series Expression Miner (STEM) has been previously described (Ernst and Bar-Joseph, 2006). Briefly, the input data table contained median FPKM expression values of all DEGs from any inter-condition comparison (2w/2 m, 2w/2y, $2 \mathrm{~m} / 2 \mathrm{y}$, FDR $\leq 0.05,-1 \geq \log _{2}$ FC $\geq 1$ ). The following parameters were utilized: log-normalization of the data, STEM clustering method, a maximum unit change in model profiles of 2, and a permutation test including time point 0 calculating all possible permutations. The integrated GO analysis tool was used. A background list of all detected genes in this sequencing dataset was provided, a minimum GO level of three selected, the minimum number of genes within a GO category set to five, and multiple hypothesis correction by randomization used.

Heatmaps are based on FPKM expression values if not stated otherwise and were generated using the online tool Morpheus (https://software.broadinstitute.org/morpheus).

\section{QUANTIFICATION AND STATISTICAL ANALYSIS}

At least 3 mice were used per experimental condition to obtain flow cytometry data. Quantification of read numbers and differential expression statistics are described above under the subheading Bioinformatic analyses. Other statistical analyses were realized using Graphpad Prism (https://www.graphpad.com/scientific-software/prism/). Diagrams are displayed as mean \pm standard deviation (SD).

\section{DATA AND CODE AVAILABILITY}

The accession number for the RNA sequencing data described in this publication is GEO: GSE133922 (https://www.ncbi.nlm.nih.gov/ geo/). 